\title{
Unicompartmental knee arthroplasty, an enigma, and the ten enigmas of medial UKA
}

\author{
Anurag Mittal ${ }^{1}$, Prashant Meshram², Woo Hyun Kim ${ }^{1}$ and Tae Kyun Kim ${ }^{1 *}$ (D)
}

\begin{abstract}
Unicompartmental knee arthroplasty (UKA) is a bone- and ligament-sparing alternative to total knee arthroplasty in the patients with end-stage single-compartment degeneration of the knee. Despite being a successful procedure, the multiple advantages of UKA do not correlate with its usage, most likely due to the concerns regarding prosthesis survivability, patient selection, ideal bearing design, and judicious use of advanced technology among many others. Therefore, the purpose of this study is to review and summarize the debated literature and discuss the controversies as "Ten Enigmas of UKA."
\end{abstract}

Keywords: Unicompartmental knee arthroplasty, UKA, Cementless, Cost effectiveness, Indications, Bearing surface

\section{Introduction}

After the discovery of Ahlback in 1968 [1] that 85\% of knees with clinical osteoarthritis (OA) have isolated medial compartment degeneration, modern modular unicompartmental knee arthroplasty (UKA) was conceptualized, thereby revolutionizing the knee arthroplasty. Since then, UKA has been an effective and minimally invasive alternative to total knee arthroplasty (TKA) that selectively replaces the damaged compartment of the knee with end-stage disease.

Historically, in 1954, McIntosh and Hunter [2] performed the first unicompartmental interpositional arthroplasty, followed by McKeever's [3] attempt of using tibial plateau prosthesis in the 1960s. In the early 1970s, St. Georg Sled [4], which was the first modular UKA, was developed by Bucholz, and ever since, the prosthetic design and its kinematics have undergone refinements with the aim of providing better clinical outcomes. Oxford knee introduction by Goodfellow and Connor [5] in the 1980s heralded a new era in UKA and has been the biggest advancement in the field of partial knee arthroplasty.

\footnotetext{
*Correspondence: osktk2000@yahoo.com

1 TK Orthopedic Surgery, 55 Dongpangyo-ro, Bundang-gu, Seongnam-si, Gyeonggi-do 13535, Republic of Korea

Full list of author information is available at the end of the article
}

Despite the newer advancements over past decades and proven advantages of minimally invasive UKA, surgeons are still reluctant to use this procedure in spite of its indication. This is most likely due to the concerns regarding survivability, patient selection, ideal bearing design, and judicious use of advanced technology among many others. The purpose of this study was to summarize the controversies pertaining to UKA as "Ten Enigmas of UKA" and review the relevant literature to highlight the available evidence related to these enigmas (Fig. 1).

1. If you had a dilemma in using UKA or TKA in an UKA-indicated patient, would you choose UKA?

2. Are classical ideal and nonideal indications of UKA proposed by Kozinn and Scott still valid with newer advanced prostheses?

3. Preoperative MRI: Do they have a role in the decision-making while considering UKA in a patient?

4. Errors in component placement: Should incision be minimal or optimal?

5. Choice of bearing design: Mobile or fixed bearing?

6. Should we use cementless implants instead of cemented ones?

7. Ideal limb alignment and optimal position of UKA prosthesis: What is the consensus?

\section{Springer Open}

(c) The Author(s) 2020. This article is licensed under a Creative Commons Attribution 4.0 International License, which permits use, sharing, adaptation, distribution and reproduction in any medium or format, as long as you give appropriate credit to the original author(s) and the source, provide a link to the Creative Commons licence, and indicate if changes were made. The images or other third party material in this article are included in the article's Creative Commons licence, unless indicated otherwise in a credit line to the material. If material is not included in the article's Creative Commons licence and your intended use is not permitted by statutory regulation or exceeds the permitted use, you will need to obtain permission directly from the copyright holder. To view a copy of this licence, visit http://creativeco mmons.org/licenses/by/4.0/. 


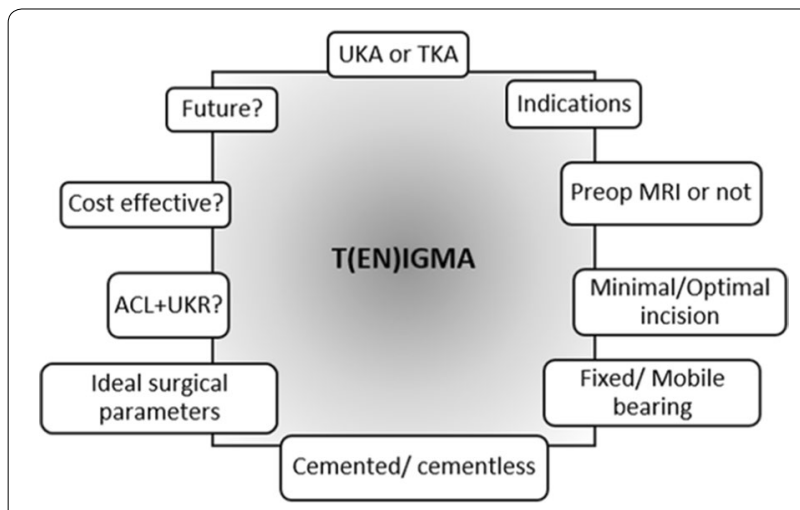

Fig. 1 Ten Enigmas (TENIGMAs) of UKA

8. Anterior cruciate ligament (ACL) reconstruction simultaneously with UKA: Is it too much?

9. Is UKA a cost-effective surgery?

10. Robotics, computer navigation, and patient-specific instrumentation and implants: Conventional versus technology-assisted UKA?

\section{If you had a dilemma in using UKA or TKA} in an UKA-indicated patient, would you choose UKA?

There is a huge burden of knee osteoarthritis in healthcare and the trends suggest that the number will continue to rise substantially due to the aging population and increased prevalence of risk factors, in particular, obesity. Subsequently, the demand for knee arthroplasty is expected to grow by more than $600 \%$ by 2030 [6]; and thus, it becomes important that patients are offered a safe and effective treatment.

UKA is considered to be a highly effective treatment after failure of conservative or joint-preserving methods for isolated osteoarthritis in the medial compartment and offers some essential short-term and long-term advantages compared with TKA. UKA has shorter operative time, reduced hospital stays, lower blood loss (reducing the number of transfusions), greater postoperative range of motion, and a higher level of activity at the time of hospital discharge [7]. Long-term benefits include preservation of bone stock for revision surgery, shorter and early recovery, lower morbidity, higher functional activity due to normal knee kinematics, and a subjective feeling of the normal knee due to preservation of the anterior and posterior cruciate ligaments and a part of the meniscus [8]. Due to extensive exposure required to perform TKA, Weale et al. observed that patella baja is more common in TKA but is rather a rare entity in UKA, which may, in part, explain the better functional results after UKA [9]. The early postoperative complications such as myocardial infarction, venous thromboembolism, cerebrovascular events, and deep infection are seen in fewer patients undergoing UKA than those undergoing TKA. Zuiderbaan et al. showed far better joint forgettability scores in UKA when compared with TKA patients [10]. Six months after surgery, Friesenbichler et al. observed that UKA patients showed better short-term quadriceps strength and gait function compared with TKA patients, together with less self-reported knee pain and stiffness [11]. Moreover, several trials report a high percentage, up to $20 \%$, of unsure or dissatisfied patients after TKA, most of them with seemingly well-fixed and well-positioned components [12].

Despite being a less invasive and having aforementioned advantages to TKA, surgeons are reluctant to consider UKA in the indicated cases. There seem to be two main reasons for the under-use of UKA. First being the reporting of a high revision rate of UKA in national joint registries (NJRs) data [13] and second being the long learning curve of UKA compared with TKA [14]. For instance, the revision rate is 3.2 times higher for UKA than TKA in the NJR for England, Wales, and Northern Ireland [15], the largest joint registry in the world. Literature indicates that around $47 \%$ of the knee osteoarthritis patients are eligible for UKA [16], however, UKA usage is a mere $5-8 \%[17,18]$. The high revision rate of UKA in NJR is in contrast with the cohort studies from highvolume centers that report similar or even better survivability of the UKA compared with TKA [19]. Also, the UKA revision rate is reported to be four times higher by lowest-volume surgeons when compared with highestvolume surgeons [20]. Thus, we envisage to comprehend the causes behind this difference and also understand the probable reasons for UKA high revision rate.

UKA is more easily revised: Knee arthroplasty surgeons are more comfortable in revising UKA than TKA because revision of an UKA tends to be less technically challenging. Evidence suggests that more tibial stems, metal augments, and stabilized components are required when a TKA is revised in comparison with a UKA [21]. Thus, the higher revision rate of UKA should not be considered a problem because it is a manifestation of an advantage of easy revision.

Lower threshold for revision of UKA than TKA: Surgeons are more likely to revise UKA than TKA even with similar Oxford knee scores (OKS). Among patients with $\mathrm{OKS}<20$ (indicating poor outcome), only $12 \%$ of TKAs were revised in contrast to $63 \%$ of UKAs with the same score [22]. So, the higher revision rate does not suggest that UKA has a worse outcome than TKA.

Selection bias: Patients selected for UKA by surgeons tend to be younger and more active, as they are the ones who meet the ideal indications for UKA surgery. 
Surgeons are worried about the progression of arthritis in the opposite compartment which is one of the main reasons for revision of an UKA. Patients with a pristine lateral and patellofemoral compartment with medial compartment arthritis are selected for UKA. However, this creates a pattern of high revision rates seen with UKA [23], younger patients naturally live longer than elderly patients, thus increasing the likelihood of future revision. Also, it has been shown that the results of arthroplasty in early arthritis lead to suboptimal results compared with arthroplasty in end-stage degeneration $[19,24]$, which can again lead to early revision. Thus, the tendency to select younger patients for UKA with less advanced osteoarthritis could lead to higher revision rate.

Lower threshold of error in implant positioning: Given that there is a longer learning curve for UKA, errors are more probable to happen in component positioning compared with TKA. Additionally, these errors are less tolerated in UKA in comparison with TKA which is somewhat forgiving in terms of alignment and softtissue balancing. Over- or under-correction of leg alignment and the tibial component malpositioning in small magnitudes are associated with an increased risk of failure. The changes from the native joint line of more than just $3^{\circ}$ in the coronal plane and $2^{\circ}$ in the sagittal plane were associated with decreased prosthesis survival [14, 25]. Thus, even if a similar error occurs while performing UKA and TKA, the UKA patient might be more likely to have an early revision.

An additional mode of failure in UKA: Apart from all the causes of revision that are similar to those of TKA, such as aseptic loosening, polyethylene wear, and tibial subsidence, there is an additional mode of failure in UKA: the progression of arthritis in unreplaced compartments [26].

Variability in UKA usage among surgeons UKA usage is defined as the proportion of patients requiring arthroplasty of the knee who are offered UKA [20]. There is a wide variation of UKA usage among surgeons, ranging from 0 to $50 \%$. Surgeons interpret the registry data and literature review about relative benefits and risks of UKA and TKA in different ways. Optimal outcomes are seen when UKA usage is between $40 \%$ and $60 \%$, whereas acceptable revision rate is achieved with a UKA usage $>20 \%$, and with a $<5 \%$ usage, there is more probability of a high revision rate for that surgeon as per the study by Liddle et al. [27]. They concluded that the proportion of UKA performed is more important than the absolute number of arthroplasties performed per year. Similarly, Hamilton et al. performed a metaanalysis on the association of caseload and usage in determining outcomes of UKA and found that usage is more important than caseload [16]. The probable reason for superior functional outcomes with increased UKA usage is the improvement in the technical and nontechnical skills (proper selection of patients and preoperative workup) of the surgeon.

As shown above, several biases and reasons lead to a perceived high revision rate of UKA. In multiple comparative studies, the Oxford knee score (OKS), the Knee Society score (KSS), and the Western Ontario and McMaster Universities Osteoarthritis index (WOMAC) indicating functional outcomes were higher following UKA than TKA [28-37] (Table 1). Thus, UKA, when indicated, could be viewed as a definitive treatment option as opposed to a first stage surgery before TKA. One of the ways to optimize clinical outcomes after UKA is to increase its usage to more than $20 \%$ for optimal results as shown by Liddle et al. [27]. This can be done by widening its indications, which brings us to our next controversy.

\section{Are classical ideal and nonideal indications of UKA proposed by Kozinn and Scott still valid with newer advanced prostheses?}

Better surgical techniques, new implant designs, improved instrumentation, and careful patient selection have led to the higher success rates of UKA in recent years [38]. Despite being a successful treatment option for medial compartment osteoarthritis, there is an ongoing debate concerning the selection criteria for UKA, as a careful patient selection is crucial to ensure excellent long-term results. Kozinn and Scott, in their landmark article of 1987, proposed the disease- and patient-specific ideal indications for UKA surgery as the isolated medial or lateral compartment osteoarthritis/osteonecrosis of the knee, age less than 60 years, weight less than $82 \mathrm{~kg}$, angular deformity less than $15^{\circ}$ (passively correctable to the neutral), flexion contracture less than $5^{\circ}$, and range of motion more than $90^{\circ}$ [39]. Patients with high activity level, exposed bone in patellofemoral compartment complaining of anterior knee pain, radiographic evidence of chondrocalcinosis, osteophytes in the opposite compartment, inflammatory arthritis, and anterior instability due to ACL insufficiency were considered nonideal candidates for partial knee replacement. Other authors have echoed this selection criteria for UKA [40]. These indications and contraindications for UKA were based on their experience only with the fixed-bearing (FB) implant and they were more intuitive than evidence based. With the advancement in prosthesis as well as surgical technique, various authors in recent studies have proposed widening the indications for UKA. They have also provided evidence of successful results with UKA performed in patients who were historically considered as "nonideal" to undergo this procedure. Pandit and colleagues, while studying 1000 patients 


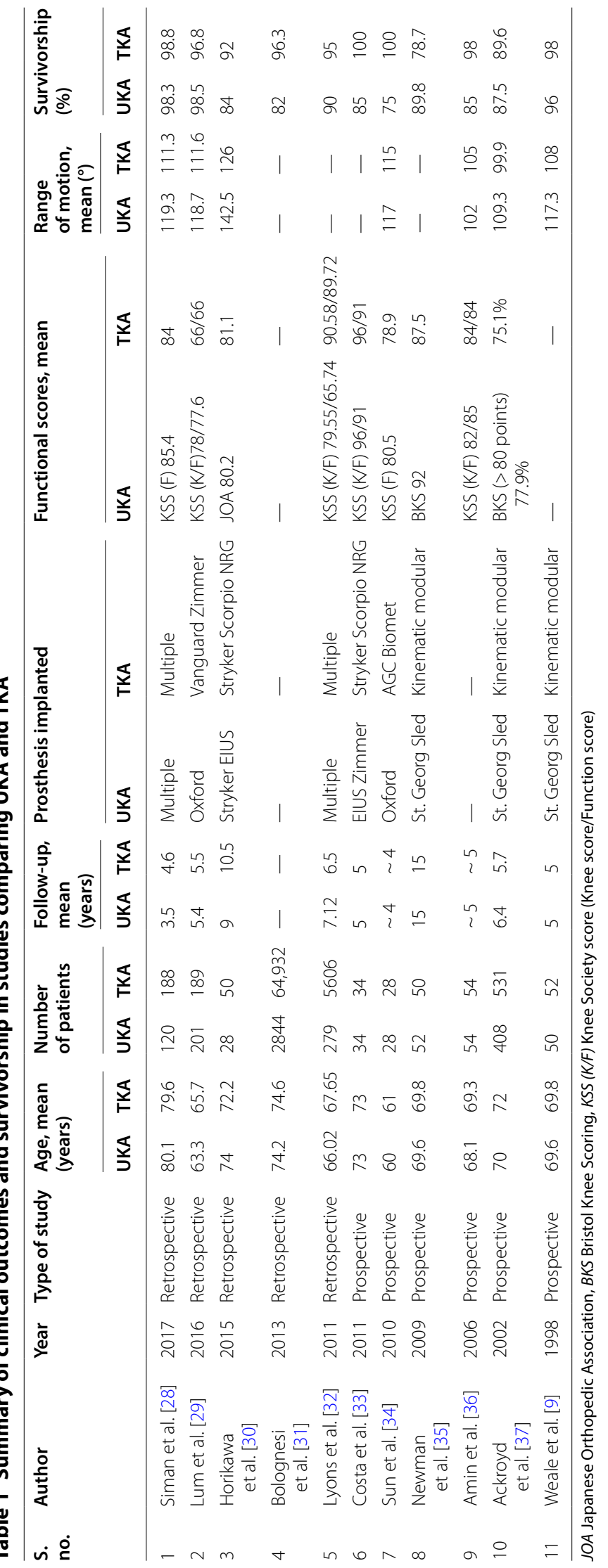


(among them, 68\% were nonideal), showed that with Oxford UKA prosthesis,10-year survival rates were similar for patients aged less than 60 years, weighing more than $82 \mathrm{~kg}$, with patellofemoral arthritis, and very active patients when compared with the ideal patient group as per Kozinn and Scott criteria [41].

UKA in patients with a high level of activity: In the next decade, knee arthroplasty utilization is expected to grow exponentially and approximately half of these procedures will be performed in patients younger than 65 years of age [42]. The high tibial osteotomy (HTO) was previously the primary means of surgical management in younger patient population with knee osteoarthritis. However, in a study by Cross et al. comparing the conversion of UKA and HTO to TKA, the complication and reoperation rates of patients in HTO group were more than twice than those in UKA group [43].

Knee arthroplasty in young patients remains a challenge for surgeons as: (i) they are often very active with high expectations concerning the ability to return to their activities after UKA, thus remaining potentially dissatisfied even after a technically successful procedure and (ii) high and regular athletic activity leads to an increase in stress on the implant-bone interface which may potentially lead to an acceleration of poly wear and early revision. Parratte et al., in their study of FB UKA performed in 35 young patients, reported a low $80.6 \%$ survival rate [44]. Similarly, Australian [45] and Swedish [46] joint registries reported the survival rate at 7 years to be $81 \%$ in patients under 55 years of age treated with UKA. Conversely, in a recent study from Germany examining return to activity in young patients following UKA, 93\% of patients returned to regular activity, and the revision rate was merely $2.5 \%$ [47]. Echoing the same, a retrospective review from Ohio done in 340 young patients aged 50 years or less showed that medial mobile-bearing UKA improved patient function and clinical parameters with a $96 \%$ survival rate free of revision surgery at 6 years and $86 \%$ at 10 years postoperatively [48]. While controversial, multiple recent studies have shown that UKA provides a viable solution for the treatment of medial compartment disease in young patient population with early patient return to function and excellent implant survival rate [41, 47, 48]. However, it is imperative that younger patients should be counseled preoperatively about potential risk for higher revision rates to set reasonable expectations from the surgery.

UKA in obese patients High body mass index (BMI) is a known risk factor for developing knee osteoarthritis, and trends towards a rising prevalence of high BMI and OA in younger patients have been documented. Conventionally, it is believed that obese patients undergoing UKA surgery tend to have poorer outcomes and early implant failure.
Berend et al., in their series of 79 patients, reported early implant failure in $22 \%$ of cases due to persistent medial pain, tibial plateau fracture, tibial loosening, and progressive arthritis [49]. Similar outcomes were reported by Peter et al. [50] and Heck et al. [51] who reported that UKA patients with a BMI greater than $32 \mathrm{~kg} / \mathrm{m}^{2}$ showed a reduced prosthesis survivorship.

As obesity has become pandemic in both developing and developed countries, a strict selection criteria based on weight is not always possible. Therefore, various studies compared the functional outcomes, survival, and complication rates of UKA in patients with normal BMI and those who were overweight and obese [52-54]. In their studies, Cavaignac [53], and Xing et al. [54] showed no adverse impact of obesity in UKA survivorship or complication rates. Tabor et al. reported higher survivorship among obese patients when compared with those who were not obese in their 20-year follow-up study of 82 patients [55]. Woo et al., in their retrospective study of 673 patients with FB UKA [56], and Molley et al., in their large prospective study of 1000 knees [57], found that high BMI is not a risk factor for loosening at a mean 10 years and there was no trend towards decreasing survival by increasing BMI. A BMI of up to $45 \mathrm{~kg} / \mathrm{m}^{2}$ is proposed as cut off below which UKA can be performed with optimal outcomes [58-60]. Thus, obesity should not be considered as a contraindication for UKA surgery. However, patients with increased weight or BMI should be counseled on the preoperative risks and the conflicting evidence regarding implant survivorship and be encouraged to lose weight to help improve this modifiable risk factor.

UKA in the presence of lateral osteophytes Osteophytes are reported to be present in $50 \%$ of knees and are considered pathognomic of osteoarthritis; however, it is unclear whether these represent localized arthritis or are a manifestation of global arthritis. Osteophytes may develop without cartilage damage owing to joint instability or lateral joint space opening. The Kellgren-Lawrence (KL) system [61] which defines osteoarthritis as "definite osteophytes with possible joint space narrowing," has been criticized in the past due to overemphasis on the presence of osteophytes. Progressive degeneration in the contralateral compartment is the most common reason for early failure of medial UKA resulting in early revision; therefore, the presence of osteophytes in the lateral compartment has been classically considered as an exclusion criterion for medial UKA, as this was believed to be associated with lateral side osteoarthritis. However, Waldstein et al. found in their study of 71 knees that lateral-compartment osteophytes are not associated with biomechanically weaker cartilage or with more advanced histologic signs of degeneration of lateral compartment 
cartilage in osteoarthritic knees with varus deformity [62]. Similarly, Faschingbauer et al., in their large series of 344 patients studied whether the presence of lateral osteophytes on plain radiographs was a predictor for quality of cartilage in the lateral compartment of patients with varus deformity and osteoarthritis (KL grade $2-3$ ). They found no difference in the cartilage thickness or cartilage volume between knees with osteophyte grades 0-3 [63].

In their clinical outcome study, Hamilton et al. followed up their UKA patients with lateral osteophytes of different Osteoarthritis Research Society International (OARSI: atlas-based grading system ranging from grade 0-no osteophytes-to grade 3-large osteophytes) grades for 15 years. Even in knees with grade 3 lateral osteophytes, there was only one failure, with a $98 \%$ survival at 15 years [64]. The authors concluded that, in knees with full-thickness cartilage in the weight-bearing portion of the lateral compartment at the time of operation, presence or severity of lateral osteophytes do not influence long-term function or implant survival following medial UKA. So, the evidence suggests that the key to the assessment of the lateral compartment is to determine the presence of full-thickness cartilage not of lateral osteophytes. Of note, the best way to assess the full-thickness cartilage in the lateral compartment is with valgus stress radiographs done preoperatively because it is difficult to assess the cartilage thickness during medial UKA surgery. Although there are no differences in cartilage thickness and volume, it is unknown whether grade 3 lateral compartment osteophytes could cause impingement when the varus deformity is reduced by medial UKA and if the probability of full-thickness cartilage defects increases significantly with the highest grade of osteophytes (grade 3).

UKA in patients with chondrocalcinosis (pseudogout) diagnosis: Chondrocalcinosis is the deposition of calcium pyrophosphate dihydrate crystal in a joint. The knee joint is the commonest site for chondrocalcinosis with a prevalence ranging from $3.2 \%$ to $6.8 \%$ [65, 66]. It is generally associated with various metabolic diseases including hyper- and hypothyroidism, hemochromatosis, gout, hypophosphatasia, hypomagnesemia, and steroid therapy [67]. There seems to be a correlation between chondrocalcinosis and osteoarthritis. If seen on radiography and during the surgery, chondrocalcinosis is considered to have elements of an inflammatory arthropathy which can lead to the progression of arthritis in the other compartments and predisposes to the development of a rapidly destructive arthropathy. However, recent evidence contradicts the theory that chondrocalcinosis predisposes to progression of arthritis in the lateral compartment among UKA patients. Only 1 case among 20 UKAs with preexisting chondrocalcinosis evaluated by Woods et al. progressed to lateral compartment osteoarthritis [68]. Hernigou et al. studied 148 UKA patients with a diagnosis of chondrocalcinosis and they had no adverse outcomes [69]. Kumar et al. reported the outcome of their consecutive series of patients with chondrocalcinosis and medial compartment osteoarthritis treated with Oxford UKA prosthesis matched to controls and encountered only one failure due to disease progression in 155 cases of chondrocalcinosis with UKA [70]. Therefore, chondrocalcinosis may not be considered as a contraindication for UKA in patients with preoperative radiological evidence.

UKA in the presence of patellofemoral joint arthritis (PFA): Due to the concerns that UKA results may be compromised, PFA has traditionally been viewed as a contraindication to UKA. This has prompted surgeons to preferentially perform TKA or combined UKA and patellofemoral arthroplasty (so-called bicompartmental knee arthroplasty) as an alternative. However, others have advocated for expanded criteria, providing evidence that utilization of UKA may be increased without compromising results even in the presence of PFA. Using the Altman classification [71], Berend et al. found no statistical difference in survivorship of UKA between patients with and without PFA, with $97.9 \%$ and $93.8 \%$ KaplanMeier survivorship at 70 months, respectively [72]. Based on their experiences with FB UKA, Thein et al. [73] and Adam et al. [74] demonstrated that preoperative patellofemoral congruence and degeneration severity without anterior knee pain do not affect postoperative functional outcomes and recommended that these patients could safely undergo FB medial UKA. Hamilton and Pandit et al. considered neither the clinical/radiological state of the PFJ nor the presence of anterior knee pain as a contraindication for UKA performed in 805 knees, except for bone loss with grooving to the lateral side [75]. In a subgroup of 100 knees, they found no relationship between functional outcomes at a mean of 10- or 15-year implant survival with the preoperative anterior knee pain, presence or degree of cartilage loss documented intraoperatively at the medial patella or trochlea, or radiographic evidence of OA in the medial side of the PFJ. Konan et al., however, found that the location of chondral lesions on the patella was an important determinant of results following UKA [76]. Centrally and laterally located chondral lesions significantly affected results and, according to the author, should be evaluated critically when considering patients with anterior knee pain and patellofemoral disease for UKA.

Patients with focal patellar PFA are indeed more likely to experience greater limitations concerning kneeling after surgery, however, we do not believe that it should be a contraindication for UKA. Firstly, though this difference 
has clinical implications regarding patient's ability to kneel, these small differences do not overshadow the significant advantages that UKA provides over multicompartmental alternatives. Secondly, according to literature, patellofemoral arthroplasty and TKA both show poorer postoperative kneeling ability compared with UKA [77]. Therefore, it is unclear whether treatment with bicompartmental knee arthroplasty or TKA would actually improve kneeling ability relative to UKA in patients with medial compartment OA and medial facet patellar degeneration. Surgeons should, however, advise patients with PFA that they may experience discomfort while kneeling. Foran et al. [78] and Berger et al. [79] have demonstrated that, despite the commonality of the development of PFA amongst patients 10-15 years after UKA, the revision rate for progressive patellofemoral arthritis is only $3 \%$. Furthermore, while the progression of arthritis in the patellofemoral compartment is common, this is rarely symptomatic. While analyzing anatomical changes following medial UKA, Thein et al. found that UKA centralizes the patellar congruence angle without impacting patellar height [73]. They proposed that restoration of patellofemoral congruence angles may release the load from the PFJ and mitigate symptoms related to patellofemoral degeneration. Therefore, patellofemoral joint osteoarthritis should not be considered as a contraindication to medial UKA, especially if the patient is asymptomatic.

In UKA, the best outcomes are achieved with proper patient selection based on its indications and contraindications. However, the above discussion derived from the plethora of evidence suggests that the thresholds proposed by Kozinn and Scott for selecting patients for UKA using weight, age, activity level, state of PFJ, and chondromalacia may not hold true. Nonetheless, if Kozinn and Scott criteria were applied, only $6-12 \%$ patients with knee osteoarthritis would be eligible for UKA; however, if expanded indications are applied, the candidacy for UKA increases to $50 \%$ [80-82]. There is an additional implication of broadening the indications of UKA as this would increase UKA usage of surgeons and as discussed earlier, could result in lower revision rates.

Goodfellow et al. recommended that indications for UKA should depend on pathoanatomy [83]. There should be substantial symptoms and anteromedial osteoarthritis/osteonecrosis, bone on bone in the medial compartment, functionally intact ACL and medial collateral ligament $(\mathrm{MCL})$, and full-thickness cartilage in the lateral compartment. Some contraindications should be adhered to such as active infection, inflammatory disease, ligamentous instability, uncorrectable varus deformity, absence of the ACL, history of HTO, and severe wear of the lateral facet of the PF joint with bone loss and grooving. These refined inclusion/exclusion criteria offer the opportunity to nearly double the number of patients who fit the criteria for medial UKA, a procedure that minimizes implant costs, surgical costs, and operative times, as well as improves patient outcomes relative to bicompartmental knee arthroplasty and TKA.

\section{Preoperative MRI: Do they have a role}

in the decision-making while considering UKA in a patient? Several radiographic studies are recommended for planning UKA including weight-bearing long-leg anteroposterior (AP) view, Rosenberg, merchant, lateral projections, and stress views. Some surgeons also prefer MRI to evaluate ligamentous structures and the lateral and patellofemoral compartment cartilage status before surgery. However, the role of preoperative MRI is debatable as it sometimes overestimates the degree of knee pathology. Disler et al. reported that nearly two-thirds of all routine knee MRIs demonstrated articular cartilage damage of uncertain clinical significance [84]. Sharpe et al. reported that, while $33 \%$ of patients with anteromedial osteoarthritis had a degenerate ACL according to MRI, only $13 \%$ patients had deficient ACL on surgical inspection. They concluded that MRI was too sensitive to be of "any practical value" in evaluating the ACL because its structure and function in osteoarthritis is highly variable [85]. In addition to the questionable utility, a routine MRI scan for planning UKA may be an avoidable expenditure [86]. Moreover, abnormal preoperative MRI findings may not influence the outcome of UKA when modern radiographic and clinical criteria are met with the appropriate intraoperative assessment. Hurst et al. reviewed 33 UKA patients who had preoperative MRI with interpretations of osteoarthritic changes in the lateral compartment, patellofemoral compartment, and/or deficiency of the ACL and reported excellent functional outcomes with only $3 \%$ failure rate [87].

Instead of a routine preoperative MRI scan, surgeons could effectively utilize physical examination for ligaments and radiographic study. Accordingly, patients with substantial ACL laxity, lateral patellar facet grooving, or collapse of the lateral compartment on valgus stress view ( $>5 \mathrm{~mm}$ narrowing of lateral joint) should not be offered UKA. Besides, UKA should be abandoned on behalf of TKA if intraoperative inspection reveals ACL incompetence or a full-thickness weight-bearing articular cartilage lesion on the lateral femoral condyle. However, surgeon reliance on the test of normal laxity of the ligament is open to error, for no quantitative measure can be reliably applied to the stretching of a ligament at surgery. Loss of height of the joint due to hyaline articular cartilage destruction leads to increased laxity of the ACL, which may in fact function normally once the articular 
height is restored by arthroplasty. Conversely, osteophyte impingement may stretch a lax ligament giving the false impression of normal tension on testing the ACL by the Lachman test. Thus, a preoperative MRI is not routinely necessary before UKA; however, when the clinical presentation is not clear, an MRI can be very useful in assessing other conditions such as avascular necrosis and neoplasm which otherwise might have gone undetected.

\section{Errors in component placement: Should incision be minimal or optimal?}

A surgical technique from its incision to the closure of the wound should be precise, safe, accurate, and reproducible. Consequently, it becomes very pertinent for any surgeon to develop evidence-based surgical protocols for favorable outcomes. Incision and exposure, the very first step of surgery, plays a vital role not only during surgery but also in rehabilitation post surgery. Conventionally, a medial parapatellar arthrotomy incision is used by most knee arthroplasty surgeons for both TKA and UKA, although the prolongation of incision proximally may be variable. Extension of the incision can be through the quadriceps tendon or the vastus medialis muscle, or a subvastus approach can be contemplated. Each approach has its advantages and disadvantages but patellar eversion is the common step which has been considered harmful. The advantages of a minimally invasive surgery (MIS) that have been reported as a reduced need for postoperative pain medication, more rapid return of knee flexion and functional activities as compared to the conventional medial parapatellar approach. Therefore, a minimally invasive incision was proposed for UKA surgery which could particularly avoid the undesirable patellar eversion. This minimally invasive approach has variations with highlights and challenges: (a) Quadriceps sparing technique with only a medial parapatellar arthrotomy without any proximal extension into the quadriceps; (b) Midvastus technique in which vastus medialis is incised for $2 \mathrm{~cm}$ along with medial parapatellar arthrotomy; and (c) Subvastus approach in which the medial parapatellar incision is prolonged by elevating the distal part of the vastus medialis muscle without performing any musculotendinous incision [88].

Haas et al. reported more rapid functional recovery with improved range of motion in TKA done with a minimidvastus approach without any malposition of implants with both standard open and mini-open surgery [89]. Price and colleagues reported the average rate of recovery after short-incision UKA to be twice as fast as that of standard-incision UKA without any significant difference in implant positioning [90]. Significant reduction in hospital bed occupancy and accelerated discharge with rapid rehabilitation resulted in $27 \%$ cost saving when mini-open surgery was compared with standard-incision UKA in a study by Reilly et al. [91]. However, some studies have raised a concern about the loss of accuracy while using the minimally invasive approach for UKA. For instance, Muller et al. comparing the two techniques in UKA surgery, found superior functional outcomes but suboptimally positioned implants with MIS than conventional incision [92]. Similarly, Dalary and Dennis showed that a significant number of patients had tibial component varus malalignment in mini-open UKA [93]. Berend and colleagues reported a $20 \%$ failure rate in short-incision UKA after follow-up of 3 years [49]. A study by Hamilton et al. observed a high revision rate and more frequent aseptic loosening for minimally invasive UKA compared with conventional UKA [94].

However, from the above discussion, it is still not clear whether to perform a minimally invasive approach or not, as the evidence is contradictory. Pragmatically, the choice of incision should depend on the preference of the surgeon and his/her expertise, as the "size" is an individual perspective. Whichever incision is chosen, this should not violate the principles of a surgical approach. Moreover, due to an increase in the life expentancy accros the globe, the probability of revision after UKA is high. Therefore, the incision should be such that future revision is comfortable. Exposure should be such that the proper landmarks for bony cuts and soft-tissue balancing are easily localized. There should be no struggle during surgery in any step as soft-tissue manhandling may inadvertently lead to poor results, which would defeat the very purpose of MIS. Complete evaluation of ACL sufficiency and the other compartment arthritis should be possible with the incision, and the surgeon should be able to work comfortably for the accomplishment of proper and complete tibial and femoral cuts with trouble-free cementation.

\section{Choice of bearing design: Mobile or fixed bearing?}

Since the introduction of UKA more than 30 years ago, the prosthesis design has significantly modified resulting in excellent functional outcomes and increased survivability. Similar to TKA, UKA surgery also has options of $\mathrm{FB}$ as well as $\mathrm{MB}$ polyethelyne designs. However, the choice of bearing design is thought to influence the functional outcomes and longevity of UKA quiet differently than in TKA. Historically, the first available UKAs were cemented FB all-polyethylene UKAs. However, due to the increased incidence of polyethylene wear, metalbacked tibia with FB polyethylene was developed. Subsequently, in 1986, Goodfellow and Connor introduced a MB UKA design to further improve upon the wear characteristic [5]. Early retrieval analysis supported this notion by showing low wear rates with a fully conforming 
MB design [95]. Each has its advantages and disadvantages, although choosing the appropriate bearing type for UKA surgery remains somewhat controversial [96].

These two designs are different in their fundamental concept [97] (Fig. 2) and in the surgical techniques required for their implantation. The MB is designed to allow more natural joint kinematics [98, 99], while also allowing a higher degree of conformity between the articular surfaces. Its distinctive design is proposed to reduce the surface and subsurface contact stresses granting less likelihood of polyethylene wear compared with FB designs $[100,101]$. An intact healthy ACL is a prerequisite for MB UKA, as there is increased stress on the ligaments. A high incidence of rupture of degenerated ACL and MCL has been reported with MB poly which results in instability of the knee [102]. Besides, MB design has a peculiar concern of bearing dislocation which can ultimately lead to early failure. The rate of bearing dislocation ranges from $0.6 \%$ to $6.5 \%$ in different studies [101, 103]. Overrelease of medial collateral ligament, undersized bearing, component malposition, and flexionextension imbalance are some of the causes of bearing dislocation [104]. Consequently, surgeons tend to keep the medial joint space of the knee tight so that bearing does not slip out. Furthermore, the surgeons tend to perfom valgus overcorrection to offload the medial compartment and further decreasing the probability of bearing dislocation and aseptic loosening [105]. Failure to do so meticulously may lead to undesirable complications of accelerated progressive lateral compartment osteoarthritis. This occurs especially when surgeons fearing bearing dislocation overcorrects the deformity into valgus alignment, leading to overt contact stresses on the lateral side $[106,107]$. Conversely, if the varus deformity is undercorrected, the chances of bearing dislocation and aseptic loosening increases [108]. Therefore, in MB design, the precise alignment and ligamentous balancing are essential to prevent bearing dislocation or impingement, which predisposes this design to be more prone to surgeon-related errors. The revision rate of $\mathrm{MB}$ prosthesis is related to UKA usage as well as the annual operation volume of the surgeon because experienced surgeons are more meticulous in maintaining the ideal surgical parameter required for pristine UKA surgery $[109,110]$.

On the other side of the spectrum is FB design. They have less conforming articular surfaces with only micromotions between the tibial baseplate and polyethylene insert. Although this type of geometry increases the point loading, there is less risk of bearing dislocation than the $\mathrm{MB}$ prosthesis. A randomized controlled study by $\mathrm{Li}$ et al. upon the in vivo kinematics of UKA did not demonstrate the proposed kinematic advantage of MB over FB, probably due to the fact that both cruciates are retained in UKA [111]. Due to higher contact stresses caused by relatively flat articular insert, the polyethylene wear rate is higher in FB than MB UKA designs [95]. However, Burton et al., in an in vitro study comparing wear rates of $\mathrm{MB}$ and $\mathrm{FB}$ designs showed reduced cumulative wear with FB UKA [112]. They found that, in both the designs, the lateral side had an increased amount of wear, suggesting that increased motion on the lateral side plays a larger role in wear generation than increased weight-bearing as seen medially.

As suggested by Zuiderbaan et al., slight varus undercorrection is desirable for optimal results in UKA surgery. As there is less likelihood of bearing dislocation in $\mathrm{FB}$, undercorrection can be attempted with these designs [113]. At the same time, avoiding overcorrection offloads the lateral compartment and may decelerate its progressive arthritis. Unlike MB designs, FB prosthesis allows loose medial joint space in UKA, which decreases the risk of aseptic loosening. At the end of FB UKA surgery, a 2-mm laxity during the valgus stress at $20^{\circ}$ flexion is the goal. The higher technical requirements and the longer learning curve needed for the surgeon in $\mathrm{MB}$ design is not required in $\mathrm{FB}$ designs, thereby making it more tolerant to surgeon-related errors and more friendly for less experienced surgeons. Regarding the incidence of radiolucent lines, studies have demonstrated an increased frequency of nonprogressive radiolucencies in MB UKA than FB design, probably because of micromotion between implant, cement surface, or both and the bone or due to overtension of the revised compartment [111]. This leads to higher chances of misinterpretation of physiological radiolucent lines resulting in erroneous revision in otherwise well-fixed UKA. While MB designs fail early due to bearing dislocation and aseptic loosening, FB design tends to fail late due to polyethylene wear and osteoarthritis progression in the nonreplaced compartment [108]. Neufield et al. reported 83\% 10-year survival of the MB compared with $90 \%$ survival for FB UKA design. Unlike FB UKA, the revision of MB design required stems or tibial augments making the reoperation more difficult [114]. Similarly, Bloom et al. reported that $46.7 \%$ of the MB UKA revisions required tibial augments compared with only $11.1 \%$ in the $\mathrm{FB}$ group [115]. MB designs can be more technically challenging, with a more pronounced learning curve, which can lead to the variability in results seen in literature, especially in studies including heterogeneous high- and low-volume centers. Bonutti et al. recommended that, low-volume surgeons are more likely to achieve predictable and high rates of survival using FB design for UKA [97].

Despite the difference in design and their mechanics, clinical outcomes and revision rates of FB and MB UKA 

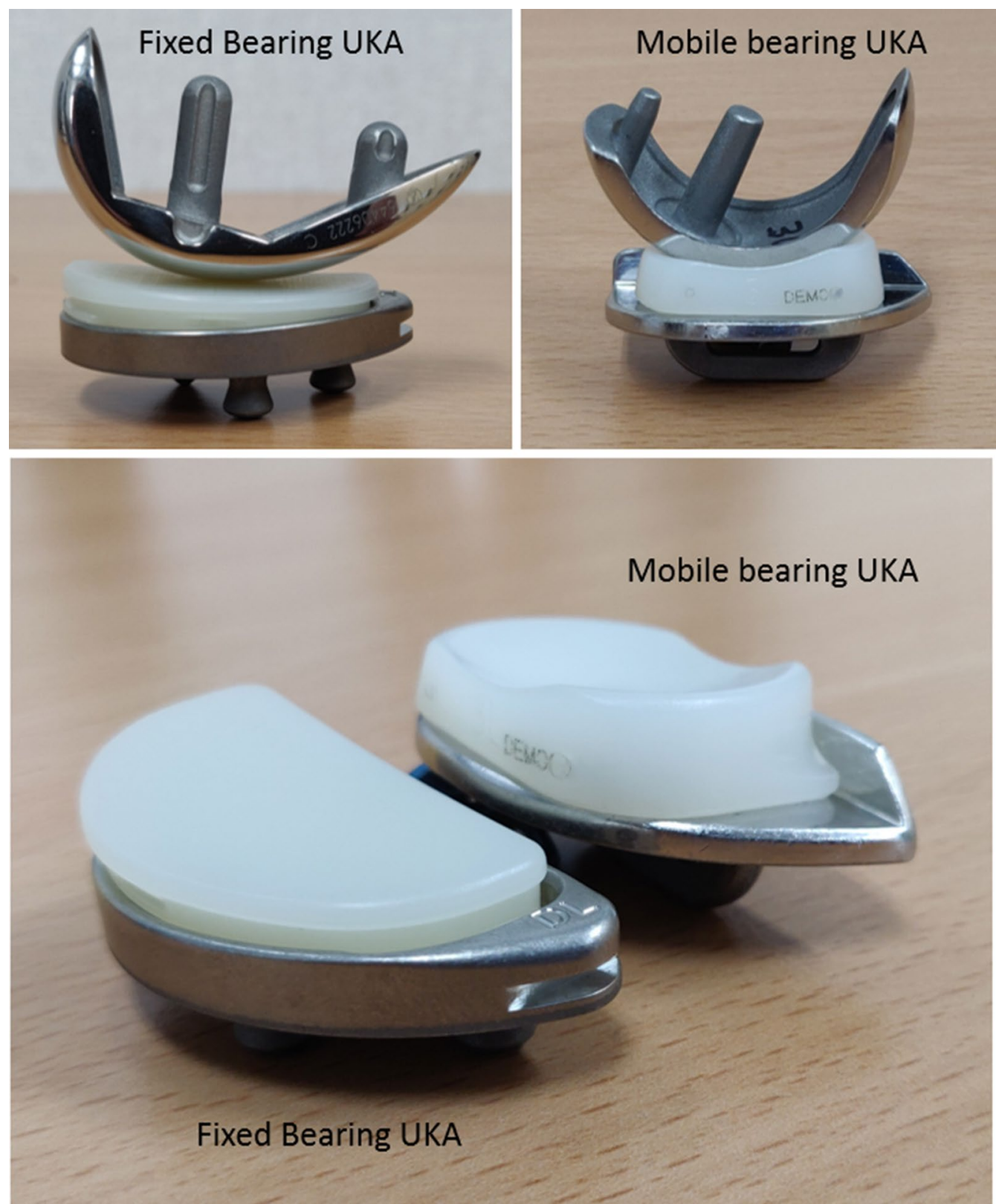

Fig. 2 Fixed bearing UKA: noncongruous articular surface, small contact area, large point contact force, and 'insert'fixed to base plate. Mobile bearing UKA: congruous articular surface, large contact area, small point contact force, and mobile 'insert'

done in primary UKA are reported as equal. Swedish [116] and Finnish [117] arthroplasty registries comparing both bearing designs of UKA suggested no conclusive advantage of one bearing design over the other in terms of prosthesis survivorship. Winnock de Grave et al. assessed 460 FB UKA and showed 94.2\% 10-year survivorship and excellent or good outcomes in $94.6 \%$ of patients [118]. Similarly, 825 MB UKA were assessed by Alnachoukati, who reported $90 \%$ 10-year survival with a mean Knee Society score of 90 postoperatively [119]. A metaanalysis by Peersman et al. also showed no differences between the two designs in terms of functional results [96]. Furthermore, a minimum 15-year follow-up long-term study by Parratte et al. observed no difference in mean Knee Society scores and survivorship between the two bearing designs [120]. A systematic review of 15 studies comparing FB and MB UKA found that there was no difference in revision rates, complications, or knee 
function [121]. The treatment option should be carefully considered for each patient and the surgeons should use their personal experience while deciding between these two bearing options.

\section{Should we use cementless implants instead of cemented ones?}

Similar to TKA, UKA can be implanted with the use of cement or using a porous-coated prosthesis surface without cement. Currently, most knee arthroplasty surgeons consider cemented UKA as gold standard despite availability of cementless implants and instrumentation, including porous-coated stem, hydroxyapatite stem fixation, and modified designs for the past 20 years. Conventional training of surgeons with cemented implants, and the high failure rates reported by a study by Lindstrand in the initial use of cementless UKA are possible reasons for underuse of cementless UKA [122].

Cementless implants have several proposed advantages over cemented implants $[123,124]$. The fixation strength in cemented prosthesis depends upon the bone ingrowth, which can lead to reliable fixation, especially in young patients. Uncemented implants eliminate the errors associated with cementation, reduced impingement [125], and no cement particulate debris which can lead to accelerated wear [126], shorter operative time, and low incidence of misinterpretation of radiolucent lines (RLLs) [127]. RLLs are prognostic factors for making a diagnosis of aseptic loosening and can be physiological or pathological. Physiological RLLs are well defined, 1-2$\mathrm{mm}$ thick, and accompanied by a radiodense line [128], in contrast to pathological RLLs that are $>2$-mm thick, poorly defined, and have no radiodense line [129]. Pandit et al. found that radiolucency occurs less frequently in uncemented UKA (6.3\% versus $75 \%$ ) in cemented UKA [127]. It has been argued that cemented UKAs show a higher incidence of radiolucencies because of the possible incomplete cementation, thermal osteonecrosis, and formation of fibrous tissue [130, 131]. Liddle et al. showed that the physiological radiolucencies are often misinterpreted on radiographs [126]. The authors defined these radiolucencies as narrow, nonprogressive, and representative of an incomplete fibrocartilage layer that does not negatively impact implant survival. In the Oxford medial UKA, the vertical wall of the tibial component is not coated with porous titanium and therefore often has adjacent radiolucencies when evaluated on radiographs postoperatively, which can be safely ignored. Thus, presence of RLLs may lead to a higher incidence of misinterpretation in cemented UKA compared with cementless prosthesis resulting in erroneous implant revisions in otherwise well-fixed and good-functioning arthroplasty.
UKA is proposed to be more befitting for cementless fixation than TKA because of the mechanical advantage of UKA at the bone-implant interface. There are mainly compressive loads both when the knee is centrally and eccentrically loaded with UKA which is an ideal condition for achieving osseous ingrowth with cementless fixation [132]. Also, shearing stress and tilting are minimal due to the absence of tibiofemoral constraints, especially in MB UKA. Liddle et al. further suggested that softtissue releases performed during routine TKA require increased tibiofemoral constraint in the form of a camand-post mechanism or dished polyethylene, which increases the shear forces imparted to the implant-bone interface and predisposes the prosthesis to aseptic loosening [126].

Daniilidis et al. while retrospectively studying 106 knees (42 cemented and 64 uncemented UKA), showed significantly better quality of life in cementless-implant patients but, at the same time, reported more and larger periprosthetic loosening areas in the cementless tibial side on radiological analysis [133]. Forsythe et al. also echoed similar concerns regarding tibial side radiolucency in cementless implants [134]. Despite early conflicting results, in the last 10 years, several specialist centers have showed encouraging clinical outcomes and survival of modern implants. Kendrick et al. in a RCT using radio isometric analysis showed no complete radiolucencies with uncemented implants, whereas $24 \%$ of cemented UKAs had complete RLLs. They concluded that the function of cementless-implant component is at least as good as if not better than that of cemented devices [124]. Pandit et al. showed no difference in clinical outcomes between the two and showed narrow RLLs at the bone-implant interfaces in $75 \%$ of cemented tibial components, whereas in the cementless implants, there was no complete radiolucency and only $7 \%$ partial lucency [127]. This implied satisfactory bone ingrowth into the cementless implants. Campi and Pandit, in their systematic review of 10 papers (1199 knees) on cementless fixation in UKA found that the 5-year survival ranged from $90 \%$ to $99 \%$ and the 10 -year survival from $92 \%$ to $97 \%$ and that the progression of osteoarthritis in the remaining compartment was the most common cause of failure [135]. Clinical outcomes, failures, reoperation rates, and survival were comparable to those reported in similar studies on cemented UKAs. This review also suggested that the robotic UKA may facilitate the implementation of cementless implants for future UKA prosthesis designs. Recent design developments, including utilizing porous titanium surfaces that allow for osseous ingrowth and coating the prosthesis with biologically active materials such as hydroxyapatite have demonstrated improved clinical and radiographic outcomes but with extra cost. 
Nonetheless, we need adequately powered RCTs and longer follow-up periods comparing cemented and cementless UKA components for reaching on a conclusive decision about superiority of clinical outcomes including survivorship.

\section{Ideal limb alignment and optimal position of UKA prosthesis: What is the consensus?}

UKA must be aimed to restore pre-osteoarthritic femorotibial geometry accurately. Several parameters have been identified as responsible for maintaining normal knee kinematics and thus avoiding common complications such as knee pain, polyethylene wear, and lateral compartment accelerated osteoarthritis in UKA. Limb alignment, component position and sizing, ligamentous and soft-tissue balancing, interprosthetic divergence, and maintenance of the inherent joint line are some of the surgical variables which have been shown to influence the clinical outcomes of UKA as well as its longevity [136] (Figs. 3, 4).

Limb alignment: The hip-knee-ankle axis (Maquet's line) determines the limb alignment and is a major contributor to a successful UKA [137]. While in TKA a neutral mechanical axis of $0^{\circ}$ is recommended, in UKA, the surgeon should correct only the part of the deformity that resulted due to wear, thus restoring the original (predisease) mechanical axis for appropriate ligament tension. Postoperative alignment in UKA is dependent on the thickness of the tibial implant, level of resection of the tibia, ligamentous balance, and preoperative deformity. Varus/valgus inclination of UKA components does not affect the lower-limb alignment but only the obliquity of the joint line. Hernigou and Deschamps while studying medial UKA, found that severe undercorrection of varus deformity could lead to accelerated polyethylene wear with early implant loosening, and at the same time, an overcorrection to valgus was found to result in rapid degeneration in the lateral compartment [138]. Zuiderbaan et al. suggested that a postoperative varus angle of $1-4^{\circ}$ should be pursued while performing medial UKA for obtaining superior functional results [139]. Similarly, Chatellard and colleagues reported high rates of mechanical failure with residual varus of $5^{\circ}$ or more [25]. Vasso and colleagues studied 125 medial FB UKA with no more than $7^{\circ}$ varus limb alignment and observed that minor varus alignment does not compromise the mid- to long-term outcomes of medial UKA and achieves better results compared with neutral alignment [140]. In general, neutral limb alignment produces good outcomes, but slight undercorrection of the initial deformity may result in the more favorable outcomes. According to few studies, a hip-knee-ankle (HKA) angle of $1-4^{\circ}$ varus optimizes subjective results of WOMAC domains of pain, function, and total scores compared with an HKA angle $<1^{\circ}$ or $>4^{\circ}[139,140]$.

Knee joint line: Restoration of the joint line in both medial and lateral compartments appears to play a role in successful outcomes following UKA. The height of the prosthetic joint space affects load transfer between the two femorotibial compartments. Finite element analysis in a previous study demonstrated increased contact stress on both the polyethylene insert and articular cartilage with a more than 6-mm change in joint line [141]. Using a validated software model for measuring joint congruence, Khamaisy et al. demonstrated that wellmaintained joint line in medial UKA improves the congruence and joint space width of the lateral compartment [142]. Elevating the medial joint line more than $5 \mathrm{~mm}$ has also been shown to result in loss of extension (flexion contracture) after UKA [143]. Mazas found that 54\% of the load is generated through medial UKA when joint space lowering is associated with $5^{\circ}$ of undercorrection. In contrast, joint space elevation associated with $5^{\circ}$ of overcorrection transferred $88 \%$ of the load to the lateral femorotibial compartment [144]. He recommended that the prosthetic joint space height should be within $3 \mathrm{~mm}$ in either direction of lateral compartment joint space height to restore balance between the two femorotibial compartments. Besides, if the transverse tibial cut is placed too distally, the tibial implant rests on cancellous bone, which offers less resistance to compressive forces, as demonstrated in an experimental study by Lesaka et al. [145]. Restoring joint space height is therefore crucial in terms of both joint mechanics and joint kinematics, as this is also responsible for limb alignment.

Tibial component position: The positioning of the prosthesis in different planes has been shown to affect the functional outcomes and the survivorship of UKA, especially the tibial component placement. In a retrospective multicentric study of 559 medial UKA, Chatellard et al. found that the tibial component obliquity is an issue that affects joint kinematics restoration and bone resistance to loading. They indicated that the physiological obliquity of the femorotibial joint space which is about $3^{\circ}$ of varus, should be restored to within $3^{\circ}$ in either direction [25]. Using radiostereometric analysis, Barbadoro et al. found that varus angulation of the tibial component $>5^{\circ}$ resulted in increased implant micromotion that could lead to loosening [146]. Collier et al. studied 245 FB UKAs and found that leaving the medial tibial plateau with varus angulation resulted in higher failure rates [147]. A normal tibial slope should be restored, as slope influences both bone quality and knee kinematics. A greater change in slope value affects the flexion range of the prosthetic knee. An excessive slope results in active anterior tibial translation, placing excessive load on the ACL. Subsequent distension 
of this ligament may result in knee instability, whereas the absence of ligament distension with load transfer to the prosthetic plateau may result in tibial-component loosening. Hernigou and Deschamps retrospectively reviewed 99 UKAs with an average follow-up of 16 years and found posterior tibial slope $>7^{\circ}$ to be associated with a higher risk of loosening [148]. Chatellard et al. identified two criteria: absolute slope should not exceed $5^{\circ}$ and the change in slope should not be greater than $2^{\circ}$ relative to the physiological value for increased longevity of UKA [25]. Sizing of the tibial component appears to matter as well. Tibial components with $>3 \mathrm{~mm}$ overhang were found to have significantly worse Oxford knee scores at 5 years after surgery, but there was no difference between implants with $<3 \mathrm{~mm}$ overhang compared with undersized components [149]. However, the authors still cautioned against significant undersizing because of the risk of subsidence and loosening.

Interprosthetic divergence: In addition to the abovedescribed criteria for tibial component positioning, the relationship between tibial and femoral components also plays a role in UKA survivorship. Divergence is influenced by both implant position and implant geometry as shown in studies of Scandinavian registries. Both components should form a $90^{\circ}$ angle with each other. Chatellard et al. indicated a margin of tolerance of $6^{\circ}$ in either direction, and they believed that intraprosthetic implant alignment depended chiefly on prosthesis design [25]. Therefore, alignment issues should be discussed in the recommendations developed by manufacturers.

\section{Role of $A C L$ deficiency in UKA; $A C L$ reconstruction simultaneously with UKA: Is it too much?}

The role of ACL functional integrity has been debated among UKA surgeons [150]. The traditional opinion is that a functionally intact ACL is a fundamental prerequisite to perform UKA [151]. Both traumatic ACL rupture and degenerative ACL deficiency can predispose to osteoarthritis in the primary knee. Patients with primary ACL rupture generally present with pain and instability and develop secondary posteromedial OA [152]. This group of young and active patients should not ideally

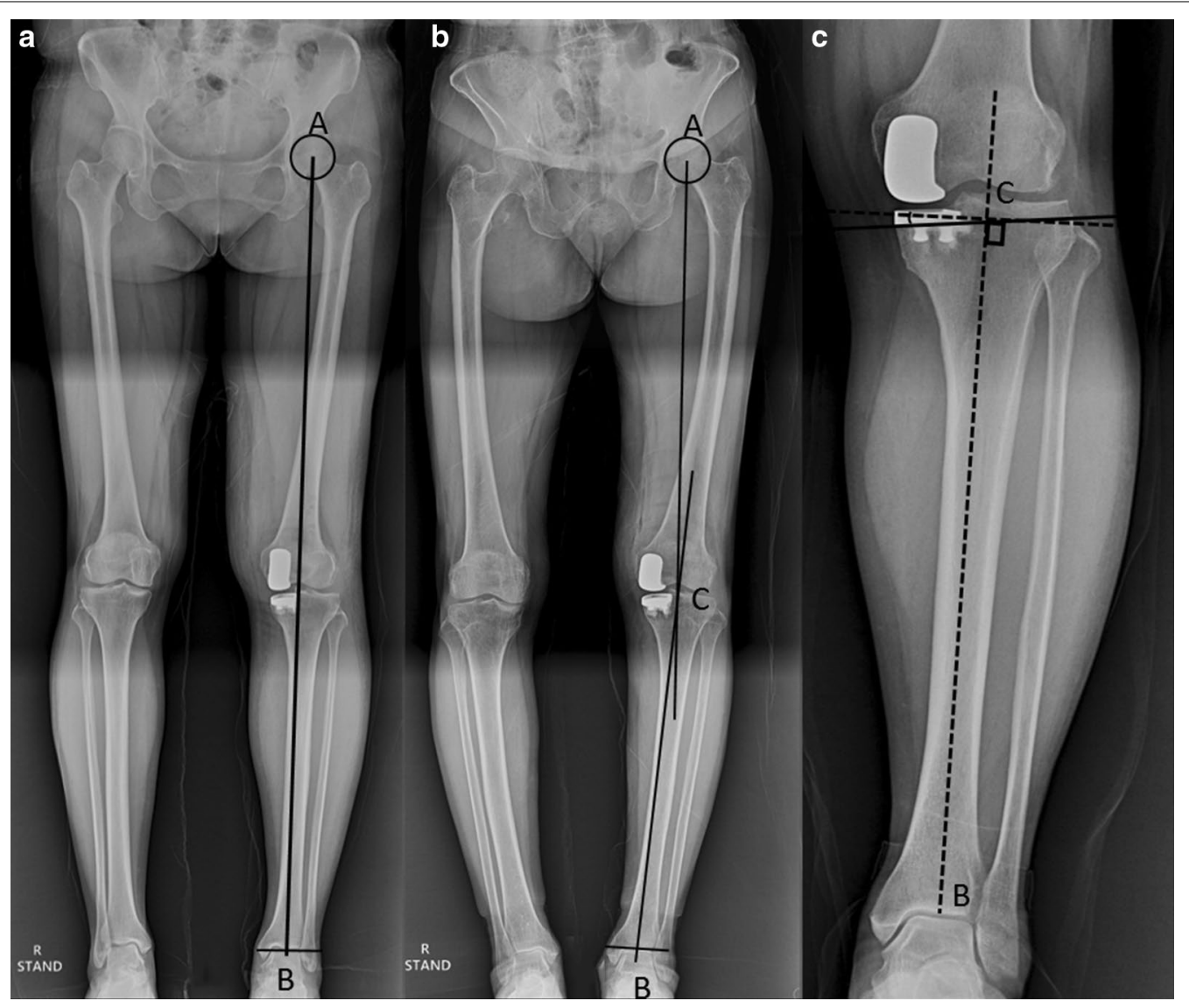

Fig. 3 a Mechanical axis (Maquet's line) is a line from center of femoral head (A) to center of ankle (B); $\mathbf{b}$ Hip-knee-ankle axis is the angle between mechanical axis of femur ( $A$ to center of knee, $C$ ) and mechanical axis of tibia ( $C$ to B); c Tibial component coronal alignment is the angle between the line perpendicular to the mechanical axis of tibia and coronal axis of tibial component 

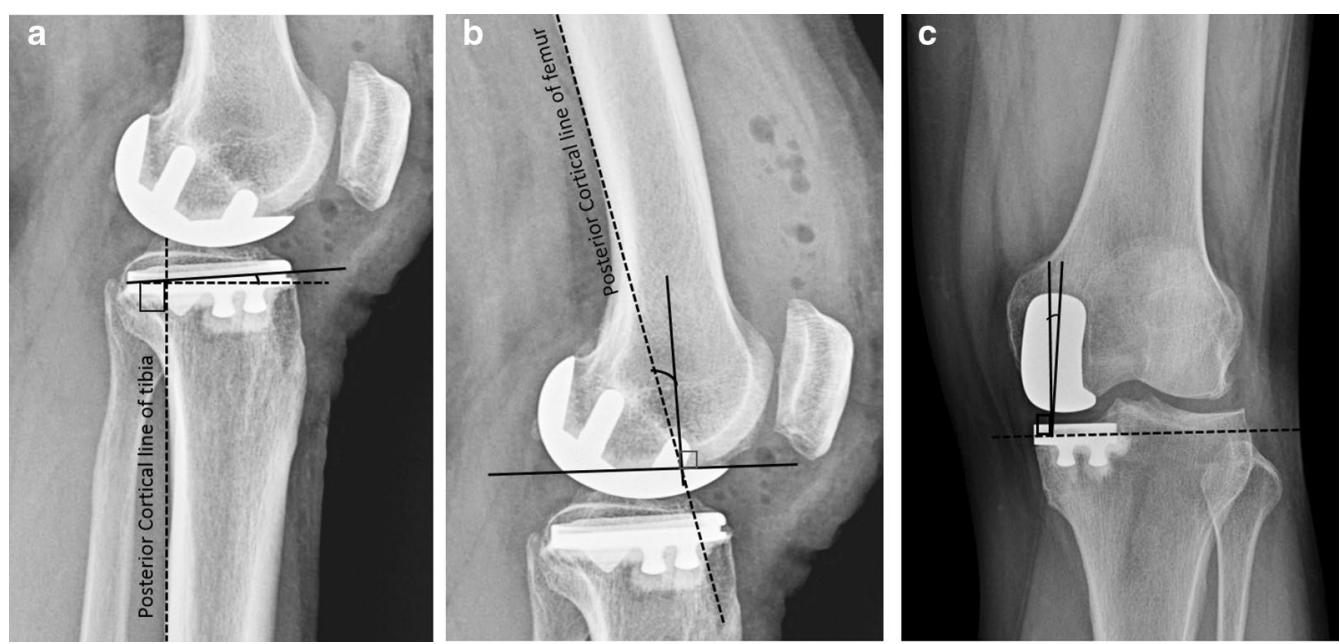

Fig. 4 a Tibial component slope is determined by the angle between the line perpendicular to posterior tibial cortex and the tibial component sagittal axis; $\mathbf{b}$ Femoral component sagittal alignment is determined by the angle between the line perpendicular to the component part placed on distal femur cut and the posterior cortical line of femur; $\mathbf{c}$ Interprosthetic divergence is the angle between the line perpendicular to the tibial component coronal axis and the long axis of femoral component

be considered candidates for isolated MB UKA because of the increased risk of failure from bearing dislocation, polyethylene wear and tibial loosening due to knee instability [153]. This is possibly related to the eccentric loading of the tibia in ACL-deficient knees. When the ACL was deficient, higher failure rates were reported with MB implants [102] and with the Lotus implant [153], a relatively flat FB component. Goodfellow et al. in their 103 MB UKA study found a 21\% failure rate within the first 2 years of implantation in ACL-deficient knees [102]. In contrast, the absence of an ACL did not lead to failure with the St. Georg and Marmor implants [151].

Elderly patients who develop secondary degenerative ACL functional deficiency due to primary anteromedial $\mathrm{OA}$ are nowadays accepted as possible candidates for UKA. Biomechanical data suggest that leveling of the tibial slope may compensate for anterior translation in the ACL-deficient knee without restoring the pivot shift to normal [154]. Also, the functional requests or the presence of posterior osteophytes and capsule stiffness prevents elderly patients from instability symptoms in most cases. Recent papers have begun to confirm good short- to mid-term outcomes without increased risk of prosthesis failure in this population. Boissonneault et al. in their small series of 46 patients with short-term follow-up and mean age of 65 years reported the 5-year survival rate for medial UKAs in ACL deficient knees to be $94 \%$ which was comparable to UKAs in ACL-intact knees [155]. ACL insufficiency being considered an absolute contraindication for UKA is debatable especially for newer FB implants as long as the degenerative pattern on the tibiofemoral joint is anterior. Notably, it is critical to minimize the tibial slope in ACL-deficient knees.

A special subgroup of patients is young individuals with rotatory ACL instability in combination with anteromedial knee OA. Technical advances and the widening of surgical indications have culminated in the advent of combined UKA and ACL reconstruction surgery which has shown promising results [156]. A review by Weston-Simons reports $93 \%$ implant survival at 5 years for 52 patients with a mean age of 51 years who underwent staged or simultaneous ACL reconstruction and $\mathrm{MB}$ UKA [157]. Combined UKA and ACL reconstruction is a longer and more technically demanding procedure but avoids the need for reoperation associated with one more anesthesia, longer recovery time, and higher social costs. A staged procedure starting with ACL reconstruction may be indicated if instability is the main symptom, proceeding with UKA only if pain arises later.

Theoretically, an absent ACL would increase the sliding motion that caused accelerated polyethylene wear of UKA in laboratory studies. However, through careful patient screening, altered intraoperative technique, and concurrent ligament reconstruction, patients with ACL-deficient knees may benefit from UKA in knees with isolated single-compartment disease. In the authors' opinion, FB UKA without ACL reconstruction can be a wiser choice in ACL-deficient knees in the lowdemand young population as well as in elderly patients. Besides, performing combined ACL reconstruction with UKA is cumbersome, requires expertise, and may result in suboptimal outcomes because of still unknown 
complications of performing ACL reconstruction simultaneously with UKA.

\section{Is UKA a cost-effective surgery?}

Joint replacement procedures including knee arthroplasty are among the most expensive procedures that are regularly performed among health-insurance beneficiaries. However, economic evaluation of any surgical procedure, if expressed in monetary terms only, would be an unfair practice. This should involve both the cost and quality-adjusted life years in the final evaluation. Therefore, effectiveness of a treatment modality should be measured in terms of (a) cost-effectiveness analysis (a common unit of clinical effect), (b) cost-utility analysis (a generic measure of health gain), and (c) cost-benefit analysis (a monetary measure) [158].

The cost of surgery and subsequent rehabilitation and reoperation is borne by either insurance company or by the patient himself depending upon the country and their health schemes. In any case, the burden of the high cost of any procedure is detrimental to the patient as well as for their country's economy. Hence, it becomes pertinent that surgeons follow a procedure that is cost-effective and at the same time, associated with satisfactory functional outcomes. UKA has been associated with a high revision rate, which can offset the initial cost-benefit gained by a shorter hospital stay and earlier rehabilitation. However, trade-offs between upfront benefits and later risk of revision of UKA compared with those of TKA are poorly understood. Konopka and his colleagues in 2008 followed up 50,493 knee replacement patients identified from the Finnish arthroplasty register and recommended discontinuation of UKA in unicompartmental OA based on low-cost effectiveness owing to high revision rates [159]. However, the analysis was not comprehensive and the study design had several methodological limitations. In contrast, recent literature without any uncertainty reports that UKA is a cost-effective procedure and should be contemplated when indicated [160, 161]. Many authors have attempted cost-effective analysis (Table 2); some are based on their literature review and their cohort analysis, and others are based on the registry data of different countries [13, 17, 159, 161-169].

The cost-effectiveness of UKA can be affected either by patient factors or the surgeon related factors. Among patient factors, age is an important variable in UKA cost-benefit analysis. Burn and Liddle in their population-based study using data from NJR from England and Wales found that the largest expected savings were for males over 75 years, while the biggest improvement in the quality of life was for females over 75 years [158]. However, it is less certain and highly variable for younger patients who have a higher risk of lifetime reoperations and revision. The variation in findings for younger patients across studies appears to be driven by differences in the estimates for both the risk of revision and the expected effect of revision on quality of life. However, modest improvements in implant survivorship could make it a cost-effective alternative in younger patients. Among surgeon related factors, surgical caseload as well as UKA usage are important considerations in the analysis of cost-effectiveness [170]. When UKA was performed by surgeons with usage above $10 \%$, UKA was found to be unequivocally cost-saving and healthimproving compared with TKA. When performed by surgeons with usage less than $10 \%$, however, UKA was no longer expected to lead to better health outcomes than TKA and TKA became the more likely cost-effective procedure [171]. UKA still compares favorably in economic evaluations of estimated cost and health outcomes even when considering slightly higher rates of revision.

\section{Robotics, computer navigation, and patient-specific instrumentation and implants: conventional versus technology-assisted UKA}

UKA, despite being associated with excellent outcomes and $96 \%$ chances of return to preoperative activity level [172], is contemplated only in 5-10\% knee arthroplasty surgeries. In contrast, TKA, which has a dissatisfaction rate of $14-19 \%$ [173] is still being ubiquitously used. UKA is a challenging procedure and outcomes after UKA patients are less tolerant to surgical errors. Execution of surgical plan intraoperatively thus becomes very important; otherwise, this may be counterproductive, as more surgeon-controlled variables have been linked to the survival of UKA. Chatellard et al. observed that a high level of accuracy is required for the optimal position of the implant and that even minute changes in the position can lead to the revision of UKA [25]. Therefore, to improve functional outcomes and address the inconsistent durability of UKA, surgeon-controlled errors must be minimum along with least outliers. There might also be a concern about the loss of accuracy with minimally invasive techniques. Consequently, assistive technologies have been developed with the potential and claims to improve the accuracy of implant positioning and limb alignment even with a minimally invasive technique by minimizing surgeon-controlled errors using smart tools.

As any industry evolves through different phases in due course of its development $[174,175]$. UKA industry, in search of an avenue for excellent outcomes and the persistent concern of its long-term survival, has integrated computer assisted technologies. These are the following: (a) computer navigation UKA, (b) patient-specific instrumentation (PSI) and implant with the help of 
three-dimensional (3D) printing, and (c) robotic-armassisted UKA (Fig. 5).

Real-time data are provided by the integration of computer technology, helping surgeons to minimize errors, especially with minimally invasive surgery. Overcorrection of limb alignment can be avoided, preventing osteoarthritis in the uninvolved compartment, thus delaying reoperation. The posterior condylar offset ratio is superior than with a conventional jig-based technique. Also, the chances of injury to essential soft tissue, especially MCL, are fewer with these newer modalities. There is higher proposed accuracy in achieving planned femoral and tibial cuts, limb alignment, soft-tissue and ligament balancing, implant position, and restoration of the native joint line. Thus, these computer assisted techniques are expected to help in the execution of surgical goals in the operating room for an even less experienced surgeon. As this eliminates the significant learning curve, inexperienced surgeons may replicate the clinical outcomes of superior function and higher longevity with UKA. Besides, the proponents of advanced technology claim improved pain control, decreased opioid analgesia dosage, and better in-patient physiotherapy than conventional UKA.

Konyves et al. revealed better implant positioning in computer-assisted navigated UKA and no difference in survivorship at 9 years compared with a conventional manual technique [176]. In a prospective study by Perlick et al., two groups of 20 UKA replacements each were operated either using a computed tomography (CT)-free navigation system or the conventional minimal invasive technique [177]. The results revealed a significant difference between the two groups in favor of navigation. In the computer-assisted group, $95 \%$ of UKAs were in a range of 4-0 degree varus (mechanical axis) compared with $70 \%$ in the conventional group. They concluded that the chances of overcorrection is diminished by realtime information about the leg axis at each step during the operation. Saragaglia et al. compared computer navigation and conventional surgery for revision of UKA to TKA [178]. They found no significant difference in the radiological target of a postoperative HKA angle at least in the hands of an experienced surgeon. However, they suggested that this technique could provide precious assistance to less experienced surgeons performing this surgery.

A prospective study of 27 patients by Cobb et al. comparing conventional jig-based UKA versus robotic UKA found that all patients undergoing robotic UKA had femorotibial axis in the coronal plane within $2^{\circ}$ of the planned position compared with only $40 \%$ in those undergoing conventional jig-based UKA [179]. Similarly, using postoperative CT scans in 62 robotic
UKAs versus 58 conventional UKAs, Bell et al. showed that robotic UKA reduced root mean square errors in achieving planned femoral and tibial implant positioning [180]. Herry et al., in their retrospective study reviewing plain radiographs of UKAs, found improved restitution of the native joint line with robotic-guided surgery compared with conventional jig-based UKAs [181].

Van den Heever et al. showed lower contact stresses and more uniform stress distribution when using patient-specific instruments and implants [182]. Jaffry et al. investigated implant positioning among PSI UKA, conventional-instrumented UKA, and roboticassisted UKA; their results showed that PSI UKA provided more accurate positioning than conventional instrumentation and no difference between roboticassisted and PSI UKA, and finally concluded that PSI UKA took half the time of robotic-assisted UKA to the implant [183]. However, Ollivier et al. concluded that claiming PSI improves alignment, pain, or function cannot justify the extra cost and uncertainty related to this technique [184]. A similar conclusion was reached by Kerens et al. comparing the radiographic positioning of implants in 30 conventional Oxford UKA with 30 patient-specific guided UKA. They found no statistically significant differences between the two groups [185].

Every new technology comes with its own set of disadvantages. There is an extra cost involved for the new high-end devices such as robotics, 3D printing, and computer navigation instruments [186]. Besides, there is an added cost of training surgery staff for these devices and instruments. Moreover, in some advanced technology, we require a preoperative CT scan for surgical planning which, apart from increasing the cost of surgery, also risks the patient's health by exposing him/her to radiation hazard. There is also a risk with the newer automated technology that the very learning curve which assistive technology seeks to circumnavigate might simply be transferred from the operating theater to the computer planning stage. In the initial stages of starting navigated or robotic-assisted UKA, there can be increased time durations for surgery, which can be frustrating for an experienced surgeon who is used to quick procedures. Additionally, the already cramped valuable space of theater gets filled up with extra logistics, instruments, and equipment. Furthermore, there is also a need to prevent surgeons from becoming technicians who are unable to independently identify and address unexpected errors in the process. Nonetheless, long-term follow-up longitudinal studies and well-planned RCTs to allow the widespread use of these new assistive technologies are still lacking. 


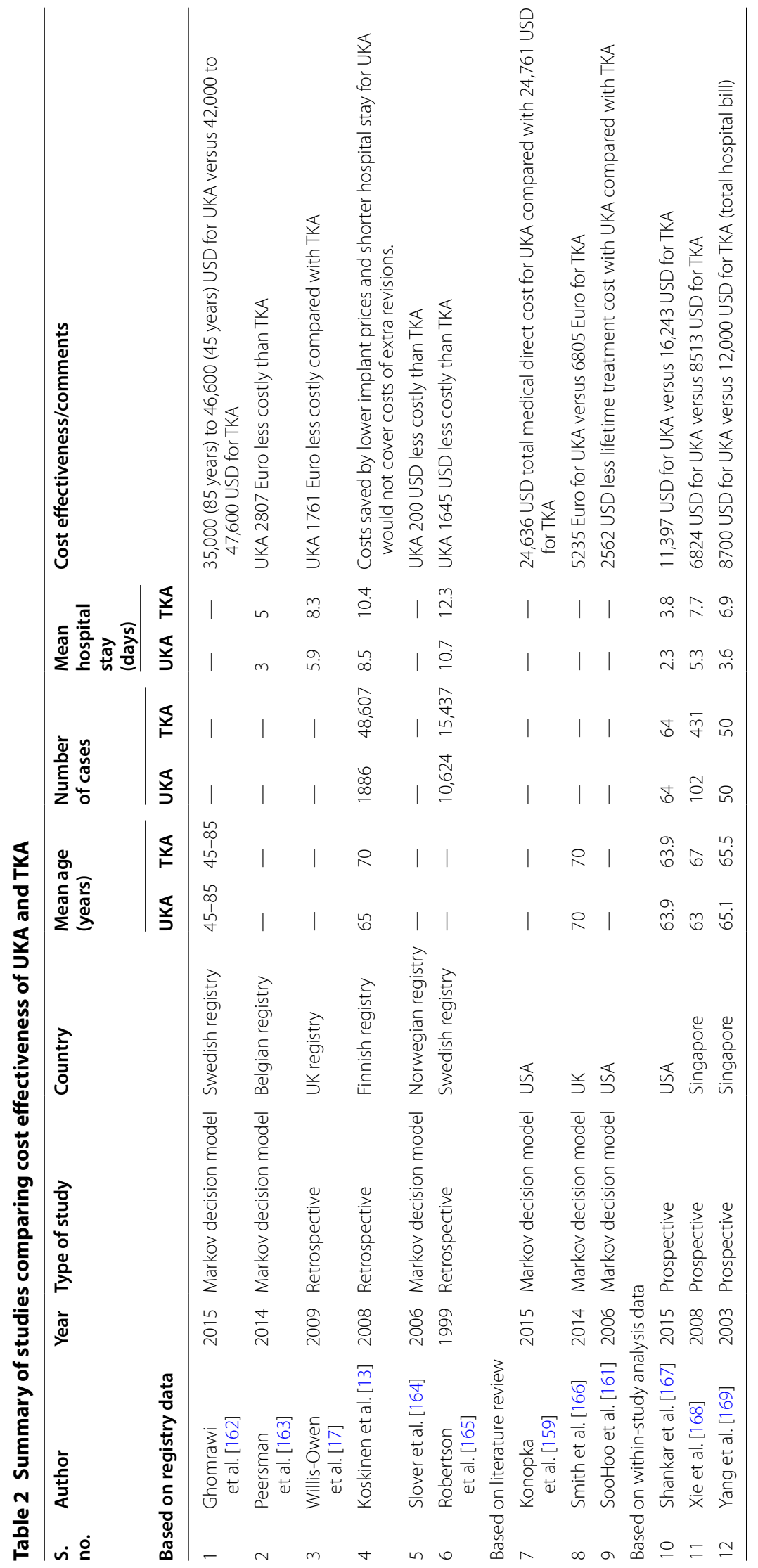




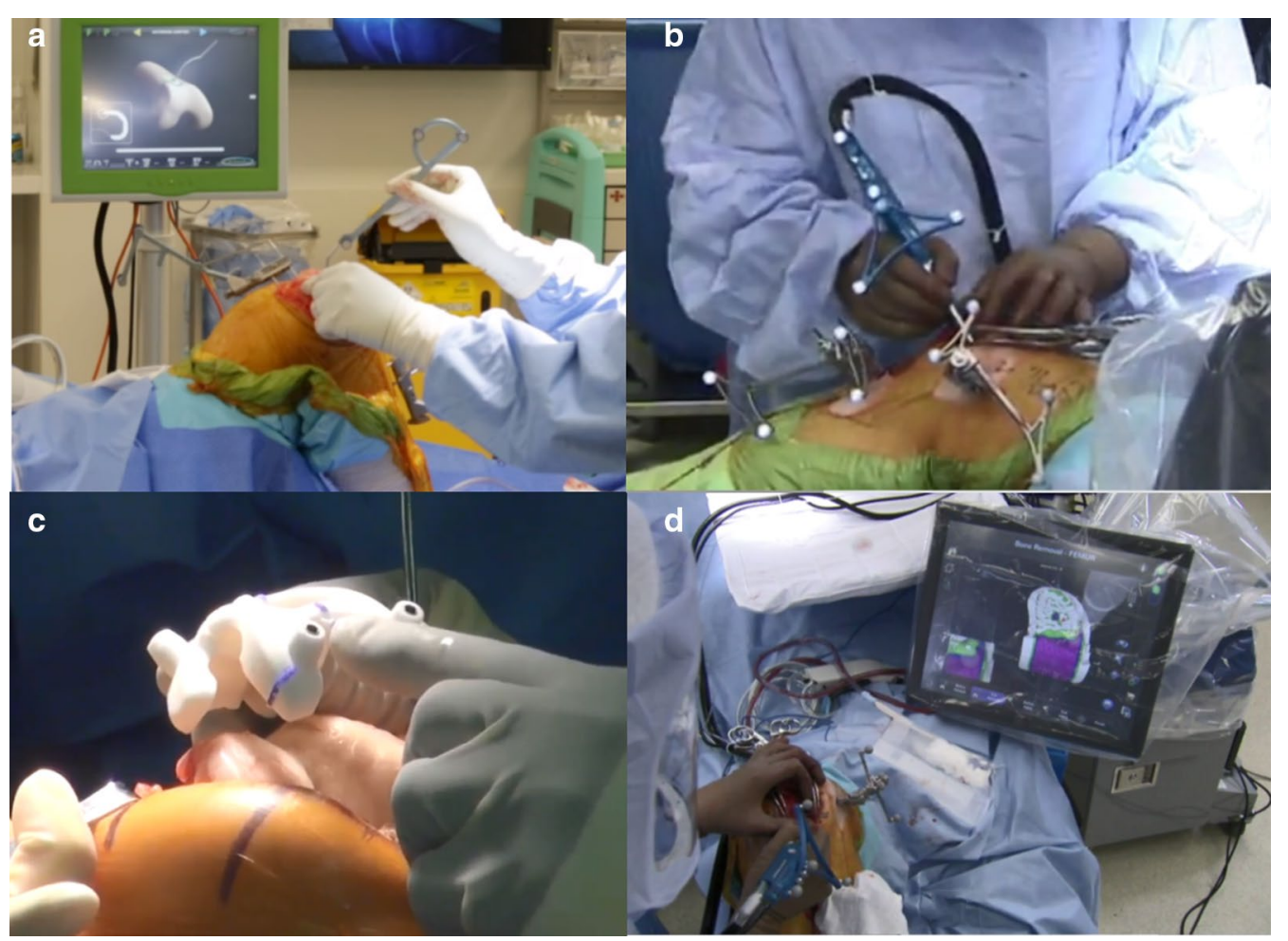

Fig. 5 a Computer Navigation, b, d Robotic-assisted UKA, c Patient-specific instrumentation (PSI)

\section{Conclusions}

The superior functional outcomes, cost-effectiveness, and improved survivorship have led to the resurgence of UKA in the past decade. Surgeons should consider UKA as a part of their armamentarium for treating knee osteoarthritis. Surgeons should aspire for optimal UKA usage in carefully selected patients along with optimal surgical technique to increase the survivability of UKA prostheses and achieve the true and full potential of UKA.

\section{Acknowledgements}

T.K.K. declares to have received, during the study period, an amount less than USD 10,000 from Smith \& Nephew (Memphis, TN) and an amount less than USD 10,000 from B. Braun (Tuttlingen, Baden-Wurttemberg, Germany) outside the submitted work. Each author declares that neither he or she nor any member of his or her immediate family has funding or commercial associations (consultancies, stock ownership, equity interest, patent/licensing arrangements, etc.) that might pose a conflict of interest in connection with the submitted article.

\section{Authors' contributions}

Research of literature and preparation of manuscript by all the authors. All authors read and approved the final manuscript.

\section{Funding}

None.

\section{Availability of data and materials} Yes.

Ethics approval and consent to participate Not required.

\section{Consent for publication}

Yes.

\section{Competing interests}

None.

\section{Author details}

${ }^{1}$ TK Orthopedic Surgery, 55 Dongpangyo-ro, Bundang-gu, Seongnam-si, Gyeonggi-do 13535, Republic of Korea. ${ }^{2}$ Department of Orthopaedics, Johns Hopkins Medical Institute, 2360 West Joppa Road, Suite 306, Baltimore, MD 21093, USA

Received: 8 March 2020 Accepted: 22 July 2020

Published online: 02 September 2020

\section{References}

1. Ahlbäck S (1968) Osteoarthrosis of the knee. A radiographic investigation. Acta Radiol Diagn (Stockh). 277:7-72

2. Maclntosh DL, Hunter GA (1972) The use of the hemiarthroplasty prosthesis for advanced osteoarthritis and rheumatoid arthritis of the knee. J Bone Joint Surg. 54(2):244-255

3. Mckeever DC (1955) The choice of prosthetic materials and evaluation of results. Clin Orthop Relat Res 6:17-21

4. Marmor L (1973) The modular knee. Clin Orthop Relat Res 94:242-248

5. Goodfellow JW, Tibrewal SB, Sherman KP, O'Connor JJ (1987) Unicompartmental oxford meniscal knee arthroplasty. J Arthroplasty 2(1):1-9

6. Kurtz S, Mowat F, Ong K et al (2005) Prevalence of primary and revision total hip and knee arthroplasty in the United States from 1990 through 2002. J Bone Joint Surg (Am) 87(A):1487-1497

7. Wiik AV, Manning V, Strachan RK, Amis AA, Cobb JP (2013) Unicompartmental knee arthroplasty enables near normal gait at higher speeds, unlike total knee arthroplasty. J Arthroplasty 28(9 Suppl):176-178 
8. HopperGP LeachWJ (2008) Participation in sporting activities following knee replacement: total versus unicompartmental. Knee Surg Sports Traumatol Arthrosc 16(10):973-979

9. Weale AE, Murray DW, Newman JH, Ackroyd CE (1999) The length of the patellar tendon after unicompartmental and total knee replacement. J Bone Joint Surg Br 81(5):790-795

10. Zuiderbaan HA, van der List JP, Khamaisy S et al (2017) Unicompartmental knee arthroplasty versus total knee arthroplasty: which type of artificial joint do patients forget? Knee Surg Sports Traumatol Arthrosc 25(3):681-686

11. Friesenbichler B et al (2018) Short-term functional advantages after medial unicompartmental versus total knee arthroplasty. Knee. https:// doi.org/10.1016/j.knee.2018.04.009

12. Dunbar MJ, Richardson G, Robertsson O (2013) I can't get no satisfaction after my total knee replacement: rhymes and reasons. Bone Joint J. 95B(11):148-152

13. Koskinen E, Eskelinen A, Paavolainen P, Pulkkinen P, Remes V (2008) Comparison of survival and cost-effectiveness between unicondylar arthroplasty and total knee arthroplasty in patients with primary osteoarthritis: a follow-up study of 50,493 knee replacements from the Finnish Arthroplasty Register. Acta Orthop 79(4):499-507

14. Zhang Q, Zhang Q, Guo W et al (2014) The learning curve for minimally invasive Oxford phase 3 unicompartmental knee arthroplasty: cumulative summation test for learning curve (LC-CUSUM). J Orthop Surg Res. 9:81. https://doi.org/10.1186/s13018-014-0081-8

15. National Joint Registry. 10th Annual Report of the National Joint Registry for England, Wales and Northern Ireland. National Joint Registry

16. Hamilton TW, Rizkalla JM, Kontochristos L et al (2017) The interaction of caseload and usage in determining outcomes of unicompartmental knee arthroplasty: a meta-analysis. J Arthroplasty. 32:3228

17. Willis-Owen CA, Brust K, Alsop H, Miraldo M, Cobb JP (2009) Unicondylar knee arthroplasty in the UK National Health Service: an analysis of candidacy, outcome and cost efficacy. Knee 16:473-478

18. Liddle AD, Judge A, Pandit H, Murray DW (2014) Adverse outcomes after total and unicompartmental knee replacement in 101,330 matched patients: a study of data from the National Joint Registry for England and Wales. Lancet 384:1437-1445

19. Pandit H, Gulati A, Jenkins C et al (2011) Unicompartmental knee replacement for patients with partial thickness cartilage loss in the affected compartment. Knee 18:168-171

20. Liddle AD, Pandit H, Judge A, Murray DW (2016) Effect of surgical caseload on revision rate following total and unicompartmental knee replacement. J Bone Joint Surg Am 98(1):1-8

21. Chawla H, van der List JP, Christ AB, Sobrero MR, Zuiderbaan HA, Pearle AD (2017) Annual revision rates of partial versus total knee arthroplasty: a comparative meta-analysis. Knee 24(2):179-190

22. Goodfellow JW, O'Connor JJ, Murray DW (2010) A critique of revision rate as an outcome measure: re-interpretation of knee joint registry data. J Bone Joint Surg Br 92(12):1628-1631

23. Murray DW, Liddle AD, Dodd CA, Pandit H (2015) Unicompartmental knee arthroplasty: is the glass half full or half empty? Bone Joint J. 97(10):3-8. https://doi.org/10.1302/0301-620x.97b10.36542

24. Niinimäki TT, Murray DW, Partanen J, Pajala A, Leppilahti Jl (2011) Unicompartmental knee arthroplasties implanted for osteoarthritis with partial loss of joint space have high re-operation rates. Knee 18:432-435

25. Chatellard R, Sauleau V, Colmar M, Robert H, Raynaud G, Brilhault J (2013) Medial unicompartmental knee arthroplasty: does tibial component position influence clinical outcomes and arthroplasty survival? Orthop Traumatol Surg Res. 99(4 SUPPL):S219-S225. https:// doi.org/10.1016/j.otsr.2013.03.004

26. Lewold S, Robertsson O, Knutson K, Lidgren L (1998) Revision of unicompartmental knee arthroplasty: outcome in 1,135 cases from the Swedish Knee Arthroplasty study. Acta Orthop Scand 69:469-474

27. Liddle AD, Pandit H, Judge A, Murray DW (2015) Optimal usage of unicompartmental knee arthroplasty: a study of 41,986 cases from the National Joint Registry for England and Wales. Bone Joint J. 97(11):1506-1511

28. Siman H, Kamath AF, Carrillo N, Harmsen WS, Pagnano MW, Sierra RJ (2017) Unicompartmental knee arthroplasty vs total knee arthroplasty for medial compartment arthritis in patients older than 75 years: comparable reoperation, revision, and complication rates. J Arthroplasty 32:1792-1797. https://doi.org/10.1016/j.arth.2017.01.020

29. Lum ZC, Lombardi AV, Hurst JM, Morris MJ, Adams JB, Berend KR (2016) Early outcomes of twin-peg mobile-bearing unicompartmental knee arthroplasty compared with primary total knee arthroplasty. Bone Joint J 98-B(Supple B):28-33. https://doi.org/10.1302/0301-620X.98B10.BJJ2016-0414.R1

30. Horikawa A, Miyakoshi N, Shimada Y, Kodama H (2015) Comparison of clinical outcomes between total knee arthroplasty and unicompartmental knee arthroplasty for osteoarthritis of the knee: a retrospective analysis of preoperative and postoperative results. J Orthop Surg Res 10:168. https://doi.org/10.1186/s13018-015-0309-2

31. Bolognesi MP, Greiner MA, Attarian DE et al (2013) Unicompartmental knee arthroplasty and total knee arthroplasty among Medicare beneficiaries, 2000 to 2009. J Bone Joint Surg Am 95:e174. https://doi. org/10.2106/JBJS.L.00652

32. Lyons MC, MacDonald SJ, Somerville LE, Naudie DD, McCalden RW (2012) Unicompartmental versus total knee arthroplasty database analysis: is there a winner? Clin Orthop Relat Res 470:84-90. https://doi. org/10.1007/s11999-011-2144-z

33. Costa CR, Johnson AJ, Mont MA, Bonutti PM (2011) Unicompartmental and total knee arthroplasty in the same patient. J Knee Surg 24:273-278. https://doi.org/10.1055/s-0031-1280970

34. Sun PF, Jia YH (2012) Mobile bearing UKA compared to fixed bearing TKA: a randomized prospective study. Knee 19:103-106. https://doi. org/10.1016/j.knee.2011.01.006

35. Newman J, Pydisetty RV, Ackroyd C (2009) Unicompartmental or total knee replacement: the 15-year results of a prospective randomised controlled trial. J Bone Joint Surg Br 91-B:52-57. https://doi. org/10.1302/0301-620x.91b1.20899

36. Amin AK, Patton JT, Cook RE, Gaston M, Brenkel IJ (2006) Unicompartmental or total knee arthroplasty?: results from a matched study. Clin Orthop Relat Res 451:101-106. https://doi.org/10.1097/01.blo.00002 24052.01873.20

37. Ackroyd CE, Whitehouse SL, Newman JH, Joslin CC (2002) A comparative study of the medial St Georg sled and kinematic total knee arthroplasties. Ten-year survivorship. J Bone Joint Surg Br 84:667-672. https:// doi.org/10.1302/0301-620x.84b5.12404

38. Price AJ, Svard U (2011) A second decade lifetable survival analysis of the Oxford unicompartmental knee arthroplasty. Clin Orthop Relat Res 469:174-179

39. Kozinn SC, Scott R (1989) Unicondylar knee arthroplasty. J Bone Joint Surg Am 71(1):145-150

40. Deshmukh RV, Scott RD (2001) Unicompartmental knee arthroplasty: long-term results. Clin Orthop Relat Res 392:272-278

41. Pandit H, Jenkins C, Gill HS, Barker K, Dodd CA, Murray DW (2011) Minimally invasive Oxford phase 3 unicompartmental knee replacement: results of 1000 cases. J Bone Joint Surg Br 93(2):198-204

42. Kurtz SM, Lau E, Ong K, Zhao K, Kelly M, Bozic KJ (2009) Future young patient demand for primary and revision joint replacement: national projections from 2010 to 2030. Clin Orthop Relat Res. 467(10):26062612 (Epub 2009 Apr 10)

43. Cross M, Yi P, Moric M (2014) Revising an HTO or UKA to TKA: is it more like a primary TKA or a Revision TKA? J Arthroplasty 229:31

44. Parratte S, Argenson J, Pearce O (2009) Medial unicompartmental knee replacement in the under-50. Bone Joint J 351:6

45. Australian Orthopaedic Association National Joint Replacement Registry (2017) Hip, Knee \& Shoulder Arthroplasty. Australian Orthopaedic Association National Joint Replacement Registry, New York

46. Dahl A, Robertsson O, Lidgren L (2010) Surgery for knee osteoarthritis in younger patients. Acta Orthop. 81(2):161-164. https://doi. org/10.3109/17453670903413186

47. Walker T, Streit J, Gotterbarm T, Bruckner T, Merle C, Streit MR (2015) Sports, physical activity and patient-reported outcomes after medial unicompartmental knee arthroplasty in young patients. J Arthroplasty 30(11):1911-1916. https://doi.org/10.1016/j.arth.2015.05.031 (Epub 2015 May 23)

48. Greco NJ, Lombardi AV Jr, Price AJ, Berend ME, Berend KR (2018) Medial mobile-bearing unicompartmental knee arthroplasty in young patients aged less than or equal to 50 years. J Arthroplasty 33(8):2435-2439. https://doi.org/10.1016/j.arth.2018.03.069 (Epub 2018 Apr 9) 
49. Berend KR, Lombardi AV Jr, Mallory TH, Adams JB, Groseth KL (2005) Early failure of minimally invasive unicompartmental knee arthroplasty is associated with obesity. Clin Orthop Relat Res 440:60-66

50. Peter MB, Maria SG, Michael GZ et al (2011) Outcomes of unicompartmental knee arthroplasty stratified by body mass index. J Arthroplasty 26(8):1149-1153

51. Heck DA, Marmor L, Gibson A et al (1993) Unicompartmental knee arthroplasty. A multicenter investigation with longterm follow-up evaluation. Clin Orthop Relat Res 286:154-159

52. Zengerink I, Duivenvoorden T, Niesten D, Verburg H, Bloem R, Mathijssen N (2015) Obesity does not influence the outcome after unicompartmental knee arthroplasty. Acta Orthop Belg 81(4):776-783

53. Cavaignac E, Lafontan V, Reina N et al (2013) Obesity has no adverse effect on the outcome of unicompartmental knee replacement at a minimum follow-up of seven years. Bone Joint J. 95(8):1064-1068

54. Xing Z, Katz J, Jiranek W (2012) Unicompartmental knee arthroplasty: factors influencing the outcome. J Knee Surg 25:369-374

55. Tabor OB Jr, Tabor OB, Bernard M, Wan JY (2005) Unicompartmental knee arthroplasty: long-term success in middle-age and obese patients. J Surg Orthop Adv 14:59-63

56. Woo YL, Chen YQ, Lai MC et al (2017) Does obesity influence early outcome of fixed- bearing unicompartmental knee arthroplasty? J Orthop Surg (Hong Kong). 25(1):2

57. Molloy J, Kennedy J, Jenkins C, Mellon S, Dodd C, Murray D (2018) Obesity should not be considered a contraindication to medial Oxford UKA: long-term patient-reported outcomes and implant survival in 1000 knees. Knee Surg Sports Traumatol Arthrosc 27:2259. https://doi. org/10.1007/s00167-018-5218-6

58. Murray DW, Goodfellow JW, Oconnor JJ (1998) The Oxford medial unicompartmental arthroplasty: a ten-year survival study. J Bone Joint Surg. Br 80:983

59. Argenson JN, O'Connor JJ (1992) Polyethylene wear in meniscal knee replacement: a one to nine-year retrieval analysis of the Oxford knee. J Bone Joint Surg. 74:228-232

60. Swienckowski JJ, Pennington DW (2004) Unicompartmental knee arthroplasty in patients sixty years of age or younger: surgical technique. J Bone Joint Surg Am. 86:131-142

61. Kellgren JH, Lawrence JS (1957) Radiological assessment of osteoarthrosis. Ann Rheum Dis 16:494-502

62. Waldstein W, Kasparek MF, Faschingbauer M, Windhager R, Boettner F (2017) Osteophytes are not associated with lateral-compartment cartilage degeneration in arthritic varus knees. Clin Orthop Relat Res. 475(5):1386-1389

63. Faschingbauer $M$, Renner $L$, Waldstein W, Boettner F (2015) Are lateral compartment osteophytes a predictor for lateral cartilage damage in varus osteoarthritic knees? Data from the Osteoarthritis Initiative. Bone Joint J. 97(12):1634-1639

64. Hamilton TW, Choudhary R, Jenkins C et al (2017) Lateral osteophytes do not represent a contraindication to medial unicompartmental knee arthroplasty: a 15-year follow-up. Knee Surg Sports Traumatol Arthrosc 25(3):652-659

65. McCarty DJ, Hogan JM, Gatter RA (1966) Studies on pathological calcifications in human cartilage. J Bone Joint Surg (Am] 48:309-325

66. Lagier R, Baud CA. Pathological calcifications of the locomotor system. In: Milhaud G, ed. IV Symposium European des Tissues Calcifies; 1968. Paris: Bouchard, 109-13

67. Dieppe PA, Calvert P (1987) Calcium pyrophosphate dihydrate deposition. Crystals and Joint Disease. Chapman \& Hall, London, pp 154-188

68. Woods DA, Wallace DA, Woods CG, McLardy-Smith P, Carr AJ, Murray DW, Martin J, Gunther T (1995) Chondrocalcinosis and medial unicompartmental knee arthroplasty. Knee 2:3

69. Hernigou P, Pascale W, Pascale V, Homma Y, Poignard A (2012) Does primary or secondary chondrocalcinosis influence long-term survivorship of unicompartmental arthroplasty? Clin Orthop Relat Res 470:1973-1979

70. Kumar V, Pandit HG, Liddle AD, Borror W, Jenkins C, Mellon SJ, Hamilton TW, Athanasou N, Dodd CA, Murray DW (2017) Comparison of outcomes after UKA in patients with and without chondrocalcinosis: a matched cohort study. Knee Surg Sports Traumatol Arthrosc 25(1):319-324. https://doi.org/10.1007/s00167-015-3578-8
71. Altman RD, Fries JF, Bloch DA et al (1987) Radiographic assessment of progression in osteoarthritis. Arthritis Rheum 30:1214-1225

72. Berend KR, Lombardi AV Jr, Morris MJ, Hurst JM, Kavolus JJ (2011) Does preoperative patellofemoral joint state affect medial unicompartmental arthroplasty survival? Orthopedics. 34(9):494-496. https://doi. org/10.3928/01477447-20110714-39

73. Thein R, Zuiderbaan HA, Khamaisy S, Nawabi DH, Poultsides LA, Pearle AD (2015) Medial unicondylar knee arthroplasty improves patellofemoral congruence: a possible mechanistic explanation for poor association between patellofemoral degeneration and clinical outcome. J Arthroplasty. 38:9

74. Adams AJ, Kazarian GS, Lonner JH (2017) Preoperative patellofemoral chondromalacia is not a contraindication for fixed-bearing medial unicompartmental knee arthroplasty. J Arthroplasty. https://doi. org/10.1016/j.arth.2017.01.002

75. Hamilton TW, Pandit HG, Maurer DG, Ostlere SJ, Jenkins C, Mellon SJ, Dodd CAF, Murray DW (2017) Anterior knee pain and evidence of osteoarthritis of the patellofemoral joint should not be considered contraindications to mobile-bearing unicompartmental knee arthroplasty: a 15-year follow-up. Bone Joint J. 99:632

76. Konan S, Haddad FS (2016) Does location of patellofemoral chondral lesion influence outcome after Oxford medial compartmental knee arthroplasty? Bone Joint J. 98(10 Supple B):11-15

77. Hassaballa MA, Porteous AJ, Newman JH, Rogers CA (2003) Can knees kneel? Kneeling ability after total, unicompartmental and patellofemoral knee arthroplasty. Knee. 10(2):155-160

78. Foran JR, Brown NM, Della Valle CJ, Berger RA, Galante JO (2013) Long-term survivorship and failure modes of unicompartmental knee arthroplasty. Clin Orthop Relat Res 471(1):102-108

79. Berger RA, Meneghini RM, Jacobs JJ, Sheinkop MB, Della Valle CJ, Rosenberg AG et al (2005) Results of unicompartmental knee arthroplasty at a minimum of ten years of follow-up. J Bone Joint Surg Am 87(5):999-1006

80. Laskin RS (2001) Unicompartmental knee replacement: some unanswered questions. Clin Orthop Relat Res 392:267-271

81. Stern SH, Becker MW, Insall JN (1993) Unicondylar knee arthroplasty. An evaluation of selection criteria. Clin Orthop Relat Res. 286:143-148

82. Berger RA, Meneghini RM, Sheinkop MB et al (2004) The progression of patellofemoral arthrosis after medial unicompartmental replacement: results at 11 to 15 years. Clin Orthop Relat Res 428:92-99

83. Goodfellow J (2006) Unicompartmental Arthroplasty with the Oxford Knee. Oxford University Press, New York

84. Disler DG, McCauley TR, Kelman CG et al (1996) Fat-suppressed threedimensional spoiled gradient-echo MR imaging of hyaline cartilage defects in the knee: comparison with standard MR imaging and arthroscopy. AJR Am J Roentgenol 167(1):127

85. Sharpe I, Tyrrell PN, White SH (2001) Magnetic resonance imaging assessment for unicompartmental knee replacement: a limited role Knee 8(3):213

86. DAMASK (Direct Access to Magnetic Resonance Imaging: Assessment for Suspect Knees) Trial Team. Cost-effectiveness of magnetic resonance imaging of the knee for patients presenting in primary care. $\mathrm{Br} J \mathrm{Gen}$ Pract 2008;58(556):e10

87. Hurst JM, Berend KR, Morris MJ, Lombardi AV Jr (2013) Abnormal preoperative MRI does not correlate with failure of UKA. J Arthroplasty 28(9 Suppl):184-186. https://doi.org/10.1016/j.arth.2013.05.011

88. Scuderi GR, Tenholder M, Capeci C (2004) Surgical approaches in miniincision total knee arthroplasty. Clin Orthop Relat Res 248:61-77

89. Haas SB, Cook S, Beksac B (2004) Minimally invasive total knee replacement through a mini midvastus approach: a comparative study. Clin Orthop Relat Res 428:68-73

90. Price AJ, Webb J, Topf H, Dodd CAF, Goodfellow JW, Murray DW (2001) Rapid recovery after Oxford unicompartmental arthroplasty through a short incision. J Arthroplasty 16:970-976

91. Reilly KA, Beard DJ, Barker KL, Dodd CA, Price AJ, Murray DW (2005) Efficacy of an accelerated recovery protocol for Oxford unicompartmental knee arthroplasty. A randomised controlled trial. Knee 12:351-357

92. Muller PE, Pellengahr C, Witt M, Kircher J, Refior HJ, Jansson V (2004) Influence of minimally invasive surgery on implant positioning and 
the functional outcome for medial unicompartmental knee arthroplasty. J Arthroplasty 19:296-301

93. Dalury DF, Dennis DA (2005) Mini-incision total knee arthroplasty can increase risk of component malalignment. Clin Orthop Relat Res 440:77-81

94. Hamilton WG, Collier MB, Tarabee E et al (2006) Incidence and reasons for reoperation after minimally invasive unicompartmental knee arthroplasty. J Arthroplasty 21 (suppl 2):98-107

95. Kwon OR, Kang KT, Son J et al (2014) Biomechanical comparison of fixed and mobile-bearing for unicomparmental knee arthroplasty using finite element analysis. J Orthop Res 32(02):338-345

96. Peersman $G$, Stuyts B, Vandenlangenbergh T, Cartier P, Fennema P (2015) Fixed-versus mobile-bearing UKA: a systematic review and meta-analysis. Knee Surg Sports Traumatol Arthrosc 23:3296-3305

97. Bonutti PM, Dethmers DA (2008) Contemporary unicompartmental knee arthroplasty: fixed versus mobile bearing. J Arthroplasty 23(7 Suppl):24-27

98. Mullaji AB, Shah S, Shetty GM (2017) Mobile-bearing medial unicompartmental knee arthroplasty restores limb alignment comparable to that of the unaffected contralateral limb. Acta Orthop 88(01):70-74

99. Choy WS, Lee KW, Kim HY, Kim KJ, Chun YS, Yang DS (2017) Mobile bearing medial unicompartmental knee arthroplasty in patients whose lifestyles involve high ${ }^{\circ}$ of knee flexion: a 10-14 year follow-up study. Knee 24(04):829-836

100. Emerson RH Jr, Hansborough T, Reitman RD, Rosenfeldt W, Higgins LL (2002) Comparison of a mobile with a fixed-bearing unicompartmental knee implant. Clin Orthop Relat Res 404:62-70

101. Whittaker JP, Naudie DD, McAuley JP, McCalden RW, MacDonald SJ, Bourne RB (2010) Does bearing design influence midterm survivorship of unicompartmental arthroplasty? Clin Orthop Relat Res 468(01):73-81

102. Goodfellow JW, Kershaw CJ, Benson DA, O'Connor MK (1988) The Oxford knee for unicompartmental osteoarthritis: the first 103 cases. J Bone Joint Surg 70:692-701

103. Murphy R, Fraser T, Mihalko WM (2015) Mobile versus fixed bearing medial unicompartmental knee arthroplasty: a series of 375 patients. Reconstr Rev 5(01):18-21

104. Huang F, Wu D, Chang J, Zhang C, Qin K, Liao F, Yin Z (2019) A comparison of mobile- and fixed-bearing unicompartmental knee arthroplasties in the treatment of medial knee osteoarthritis: a systematic review and meta-analysis of 1,861 patients. J Knee Surg. https://doi. org/10.1055/s-0039-1697901

105. Peersman G, Slane J, Vuylsteke P, Fuchs-Winkelmann S, Dworschak P, Heyse T et al (2017) Kinematics of mobile-bearing unicompartmental knee arthroplasty compared to native: results from an in vitro study. Arch Orthop Trauma Surg 137:1557e63

106. Ko YB, Gujarathi MR, Oh KJ (2015) Outcome of unicompartmental knee arthroplasty: a systematic review of comparative studies between fixed and mobile bearings focusing on complications. Knee Surg Relat Res 27:141-148

107. Smith TO, Hing CB, Davies L, Donell ST (2009) Fixed versus mobile bearing unicompartmental knee replacement: a meta-analysis. Orthop Traumatol Surg Res 95:599-605

108. van der List JP, Zuiderbaan HA, Pearle AD (2016) Why do medial unicompartmental knee arthroplasties fail today? J Arthroplasty 31:1016-1021

109. Rajasekhar C, Das S, Smith A (2004) Unicompartmental knee arthroplasty 2- to 12-year results in a community hospital. J Bone Joint Surg $\mathrm{Br}$ 86:983-985

110. Robertsson O, Knutson K, Lewold S, Lidgren L (2001) The routine of surgical management reduces failure after unicompartmental knee arthroplasty. J Bone Joint Surg Br 83:45-49

111. Li MG, Yao F, Joss B, loppolo J, Nivbrant B, Wood D (2006) Mobile vs fixed bearing unicondylar knee arthroplasty: a randomized study on short term clinical outcomes and knee kinematics. Knee 13(05):365-370

112. Burton A, Williams S, Brockett CL, Fisher J (2012) In vitro comparison of fixed- and mobile meniscal-bearing unicondylar knee arthroplasties: effect of design, kinematics, and condylar liftoff. J Arthroplasty 27(8):1452-1459. https://doi.org/10.1016/j.arth.2012.02.011 (Epub 2012 Apr 11)
113. Zuiderbaan HA, van der List JP, Kleeblad LJ et al (2016) Modern indications, results, and global trends in the use of unicompartmental knee arthroplasty and high tibial osteotomy in the treatment of isolated medial compartment osteoarthritis. Am J Orthop 45(06):E355-E361

114. Neufeld ME, Albers A, Greidanus NV, Garbuz DS, Masri BA (2018) A comparison of mobile and fixed-bearing unicompartmental knee arthroplasty at a minimum 10-year follow-up. J Arthroplasty 33(06):1713-1718

115. Bloom KJ, Gupta RR, Caravella JW, Shishani YF, Klika AK, Barsoum WK (2014) The effects of primary implant bearing design on the complexity of revision unicondylar knee arthroplasty. J Arthroplasty 29:106-109. https://doi.org/10.1016/j.arth.2013.04.021

116. Lewold S, Goodman S, Knutson K, Robertsson O, Lidgren L (1995) Oxford meniscal bearing knee versus the Marmor knee in unicompartmental arthroplasty for arthrosis. A Swedish multicenter survival study. J Arthroplasty 10(6):722-731

117. Koskinen E, Paavolainen P, Eskelinen A, Pulkkinen P, Remes V (2007) Unicondylar knee replacement for primary osteoarthritis: a prospective follow-up study of 1,819 patients from the Finnish Arthroplasty Register. Acta Orthop 78:128-135

118. Winnock de Grave P, Barbier J, Luyckx T, Ryckaert A, Gunst P, Van den Daelen L (2018) Outcomes of a fixed-bearing, medial, cemented unicondylar knee arthroplasty design: survival analysis and functional score of 460 cases. J Arthroplasty 33:2792-2799

119. Alnachoukati OK, Barrington JW, Berend KR, Kolczun MC, Emerson RH, Lombardi AV Jr et al (2018) Eight hundred twenty-five medial mobilebearing unicompartmental knee arthroplasties: the first 10-year US multi-center survival analysis. J Arthroplasty 33:677-683

120. Parratte S, Pauly V, Aubaniac JM, Argenson JN (2012) No long-term difference between fixed and mobile medial unicompartmental arthroplasty. Clin Orthop Relat Res 470(01):61-68

121. Cao Z, Niu C, Gong C, Sun Y, Xie J, Song Y (2019) Comparison of fixedbearing and mobile-bearing unicompartmental knee arthroplasty: a systematic review and meta-analysis. J Arthroplasty. 34(12):3114-3123. https://doi.org/10.1016/j.arth.2019.07.005 (Epub 2019 Jul 30)

122. Lindstrand A, Stenstrom A, Egund N (1988) The PCA unicompartmental knee A 1-4-year comparison of fixation with or without cement. Acta Orthop Scand 59(6):695-700

123. Hooper N, Snell D, Hooper G, Maxwell R, Frampton C (2015) The fiveyear radiological results of the uncemented Oxford medial compartment knee arthroplasty. Bone Joint J 97:1358-1363

124. Kendrick BJL, Kaptein BL, Valstar ER, Gill HS, Jackson WFM, Dodd CAF, Price AJ, Murray DW (2015) Cemented versus cementless Oxford unicompartmental knee arthroplasty using radiostereometric analysis: a randomised controlled trial. Bone Joint J 97:185-191

125. Liddle AD, Pandit H, O'Brien S, Doran E, Penny ID, Hooper GJ, Burn PJ, Dodd CAF, Beverland DE, Maxwell AR, Murray DW (2013) Cementless fixation in Oxford unicompartmental knee replacement: a multicentre study of 1000 knees. Bone Joint J 95:181-187

126. Liddle AD, Pandit H, Murray DW, Dodd CaF (2013) Cementless unicondylar knee arthroplasty. Orthop Clin North Am 44:261-269

127. Pandit H, Jenkins C, Beard DJ, Gallagher J, Price AJ, Dodd CaF, Goodfellow JW, Murray DW (2009) Cementless Oxford unicompartmental knee replacement shows reduced radiolucency at one year. J Bone Joint Surg $\operatorname{Br}$ 91:185-189

128. Gulati A, Chau R, Pandit HG, Gray H, Price AJ, Dodd CA, Murray DW (2009) The incidence of physiological radiolucency following Oxford unicompartmental knee replacement and its relationship to outcome. $J$ Bone Joint Surg Br 91(7):896-902

129. Mukherjee K, Pandit H, Dcdd CA, Ostlere S, Murray DW (2008) The Oxford uniccmpartmental knee arthroplasty: a radiological perspective. Clin Radicl. 63(10):1169-1176

130. Kendrick BJL, James AR, Pandit H, Gill HS, Price AJ, Blunn GW et al (2012) Histology of the bone-cement interface in retrieved Oxford unicompartmental knee replacements. Knee 19:918-922

131. Windsor RE, Scuderi GR, Moran MC, Insall JN (1989) Mechanisms of failure of the femoral and tibial components in total knee arthroplasty. Clin Orthop Relat Res 248:15-20

132. O'Connor JJ, Goodfellow JW (1996) Theory and practice of meniscal knee replacement: designing against wear. Proc Inst Mech Eng $\mathrm{H}$. 210(3):217-222 
133. Daniilidis K, Skwara A, Skuginna A, Fischer F, Tibesku CO. [Comparison of medium-term clinical and radiological outcome between cemented and cementless medial unicompartmental knee arthroplasty]. Z Orthop Unfall. 2009;147(2):188-93. https://doi. org/10.1055/s-0029-1185528. Epub 2009 Apr 8. German

134. Forsythe ME, Englund RE, Leigton RK (2000) Unicondylar knee arthroplasty: a cementless perspective. Can J Surg 43(6):417-424

135. Campi S, Pandit HG, Dodd CAF, Murray DW (2017) Cementless fixation in medial unicompartmental knee arthroplasty: a systematic review. Knee Surg Sports Traumatol Arthrosc 25(3):736-745

136. Walker T, Zahn N, Bruckner T et al (2018) Mid-term results of lateral unicondylar mobile bearing knee arthroplasty: a multicentre study of 363 cases. Bone Joint J 100:42-49

137. Kennedy WR, White RP (1987) Unicompartmental arthroplasty of the knee: postoperative alignment and its influence on overall results. Clin Orthop 221:278-285

138. Hernigou P, Deschamps G (2004) alignment influences wear in the knee after medial unicompartmental arthroplasty. Clin Orthop 423:161-165

139. Zuiderbaan HA, van der List JP, Chawla H, Khamaisy S, Thein R, Pearle AD (2016) Predictors of subjective outcome after medial unicompartmental knee arthroplasty. J Arthroplasty 31(7):1453-1458. https://doi. org/10.1016/j.arth.2015.12.038 (Epub 2016 Feb 28)

140. Vasso M, Del Regno C, D'Amelio A, Viggiano D, Corona K, Schiavone Panni A (2015) Minor varus alignment provides better results than neutral alignment in medial UKA. Knee. 22(2):117-121. https://doi. org/10.1016/j.knee.2014.12.004 (Epub 2014 Dec 13)

141. Kwon OR, Kang KT, Son J, Suh DS, Baek C, Koh YG (2017) Importance of joint line preservation in unicompartmental knee arthroplasty: finite element analysis. J Orthop Res 35(2):347-352. https://doi. org/10.1002/jor.23279

142. Khamaisy S, Zuiderbaan HA, van der List JP, Nam D, Pearle AD (2016) Medial unicompartmental knee arthroplasty improves congruence and restores joint space width of the lateral compartment. Knee. 23(3):501-505. https://doi.org/10.1016/j.knee.2016.02.012 (Epub

\section{Mar 16)}

143. Takayama K, Ishida K, Muratsu H, Kuroda Y, Tsubosaka M, Hashimoto S, Hayashi S, Matsushita T, Niikura T, Kuroda R, Matsumoto T (2018) The medial tibial joint line elevation over $5 \mathrm{~mm}$ restrained the improvement of knee extension angle in unicompartmental knee arthroplasty. Knee Surg Sports Traumatol Arthrosc 26(6):1737-1742. https://doi.org/10.1007/s00167-017-4763-8

144. Mazas F. Arthroplastie unicompartimentaire du genou. Cahiers d'enseignement de la SOFCOT no 34. Paris: Elsevier Masson; 1989. p. 113-23

145. Lesaka K, Tsumura H, Sonoda H, Sawatari T, Takasita M, Torisu T (2002) The effects of tibial component inclination on bone stress after unicompartmental knee arthroplasty. J Biomech 35(7):969-974

146. Barbadoro P, Ensini A, Leardini A, d'Amato M, Feliciangeli A, Timoncini A, Amadei F, Belvedere C, Giannini S (2014) Tibial component alignment and risk of loosening in unicompartmental knee arthroplasty: a radiographic and radiostereometric study. Knee Surg Sports Traumatol Arthrosc 22(12):3157-3162. https://doi.org/10.1007/s0016 7-014-3147-6 (Epub 2014 Jun 28)

147. Collier MB, Eickmann TH, Sukezaki F, McAuley JP, Engh GA (2006) Patient, implant, and alignment factors associated with revision of medial compartment unicondylar arthroplasty. J Arthroplasty 21:108-115

148. Hernigou P, Deschamps G (2004) Posterior slope of the tibial implant and the outcome of unicompartmental knee arthroplasty. J Bone Joint Surg Am 86(3):506-511

149. Chau R, Gulati A, Pandit H, Beard DJ, Price AJ, Dodd CA, Gill HS, Murray DW (2009) Tibial component overhang following unicompartmental knee replacement-does it matter? Knee. 16(5):310-313. https://doi. org/10.1016/j.knee.2008.12.017

150. Engh GA, Ammeen D (2004) Is an intact anterior cruciate ligmament needed in order to have a well-functioning unicondylar knee arthroplasty? Clin Orthop Relat Res 428:170-173

151. Cartier P, Sanouiller JL, Greslamer RP (1996) Unicompartmental knee arthroplasty surgery. 10-year minimum follow-up period. J Arthroplasty 11:782-788
152. Pandit H, Beard J, Jenkins C, Kimstra Y, Thomas NP, Dodd CAF, Murray DW (2006) Combined anterior cruciate reconstruction and Oxford unicompartmental knee arthoplasty. J Bone Joint Surg 88:887-892

153. Deschamps G, Lapeyre B (1987) Rupture of the anterior cruciate ligament: a frequently unrecognized cause of failure of unicompartmental knee prostheses: apropos of a series of 79 Lotus prostheses with a follow-up of more than 5 years. Rev Chir Orthop Reparatrice Appar Mot 73:544-551 (in French)

154. Suero EM, Citak M, Cross MB, Bosscher MRF, Ranawat AS, Pearle AD (2012) Effects of tibial slope changes in the stability of fixed bearing medial unicompartmental arthroplasty in anterior cruciate ligament deficient knees. Knee. 19:365-369

155. Boissonneault A, Pandit H, Pegg E, Jenkins C, Gill HS, Dodd CA, Gibbons CL, Murray DW (2013) No difference in survivorship after unicompartmental knee arthroplasty with or without an intact anterior cruciate ligament. Knee Surg Sports Traumatol Arthrosc 21(11):2480-2486. https ://doi.org/10.1007/s00167-012-2101-8 (Epub 2012 Jul 11)

156. Tinius M, Hepp P, Becker R (2012) Combined unicompartmental knee arthroplasty and anterior cruciate ligament reconstruction. Knee Surg Sports Traumatol Arthrosc 20:81-87

157. Weston-Simons JS, Pandit H, Jenkins C, Jackson WF, Price AJ, Gill HS, Dodd CA, Murray DW (2012) Outcome of combined unicompartmental knee replacement and combined or sequential anterior cruciate ligament reconstruction: a study of 52 cases with mean follow-up of five years. J Bone Joint Surg Br 94(9):1216-1220. https://doi. org/10.1302/0301-620X.94B9.28881

158. Burn E, Liddle AD, Hamilton TW, Pai S, Pandit HG, Murray DW, PinedoVillanueva R (2017) Choosing Between Unicompartmental and Total Knee Replacement: what Can Economic Evaluations Tell Us? A Systematic Review. Pharmacoecon Open. 1(4):241-253. https://doi. org/10.1007/s41669-017-0017-4

159. Konopka JF, Gomoll AH, Thornhill TS, Katz JN, Losina E (2015) The costeffectiveness of surgical treatment of medial unicompartmental knee osteoarthritis in younger patients: a computer modelbased evaluation. J Bone Jt Surg Am. 97(10):807-817

160. Manzotti A, Confalonieri N, Pullen C (2007) Unicompartmental versus computer-assisted total knee replacement for medial compartment knee arthritis: a matched paired study. Int Orthop 31(3):315-319

161. Soohoo NF, Sharifi H, Kominski G, Lieberman JR (2006) Cost-effectiveness analysis of unicompartmental knee arthroplasty as an alternative to total knee arthroplasty for unicompartmental osteoarthritis. J Bone Jt Surg Am. 88(9):1975-1982

162. Ghomrawi HM, Eggman AA, Pearle AD (2015) Effect of age on costeffectiveness of unicompartmental knee arthroplasty compared with total knee arthroplasty in the US. J Bone Jt Surg Am. 97(5):396-402

163. Peersman G, Jak W, Vandenlangenbergh T, Jans C, Cartier P, Fennema P (2014) Cost-effectiveness of unicondylar versus total knee arthroplasty: a Markov model analysis. Knee. 21:S37-S42

164. Slover J, Espehaug B, Havelin LI, Engesaeter LB, Furnes O, Tomek I et al (2006) Cost-effectiveness of unicompartmental and total knee arthroplasty in elderly low-demand patients. A Markov decision analysis. J Bone Jt Surg Am. 88(11):2348-2355

165. Robertsson O, Borgquist L, Knutson K, Lewold S, Lidgren L (1999) Use of unicompartmental instead of tricompart-mental prostheses for unicompartmental arthrosis in the knee is a cost-effective alternative: 15,437 primary tricompartmental prostheses were compared with 10,624 primary medial or lateral unicompartmental prostheses. Acta Orthopaed. 70(2):170-175

166. Smith WB 2nd, Steinberg J, Scholtes S, Mcnamara IR (2017) Medial compartment knee osteoarthritis: age-stratified cost-effectiveness of total knee arthroplasty, unicompartmental knee arthroplasty, and high tibial osteotomy. Knee Surg Sports Traumatol Arthrosc 25(3):924-933. https://doi.org/10.1007/s00167-015-3821-3

167. Shankar S, Tetreault MW, Jegier BJ, Andersson GB, Della Valle CJ (2016) A cost comparison of unicompartmental and total knee arthroplasty. Knee. 23(6):1016-1019. https://doi.org/10.1016/j.knee.2015.11.012

168. Xie F, Lo NN, Tarride JE, O'Reilly D, Goeree R, Lee HP (2010) Total or partial knee replacement? Cost-utility analysis in patients with knee osteoarthritis based on a 2-year observational study. Eur J Health Econ. $11(1): 27-34$ 
169. Yang KY, Wang MC, Yeo SJ, Lo NN (2003) Minimally invasive unicondylar versus total condylar knee arthroplasty: early results of a matched-pair comparison. Singap Med J. 44:559-562

170. Burn E, Sanchez-Santos MT, Pandit HG, Hamilton TW, Liddle AD, Murray DW, et al. Ten-year patient-reported outcomes following total and minimally invasive unicompartmental knee arthroplasty: a propensity score-matched cohort analysis. Knee Surg Sports Traumatol Arthrosc. (Epub 29 Dec 2016).

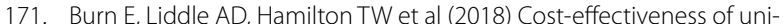
compartmental compared with total knee replacement: a populationbased study using data from the National Joint Registry for England and Wales. BMJ Open 8:e020977. https://doi.org/10.1136/bmjop en-2017-020977

172. Witjes S, Gouttebarge V, Kuijer PP, van Geenen RC, Poolman RW, Kerkhoffs GM (2016) Return to sports and physical activity after total and unicondylar knee arthroplasty: a systematic review and meta-analysis. Sports Med 46(2):269-292. https://doi.org/10.1007/s40279-015-0421-9

173. Bourne RB, Chesworth BM, Davis AM, Mahomed NN, Charron KD (2010) Patient satisfaction after total knee arthroplasty: who is satisfied and who is not? Clin Orthop Relat Res 468:57-63

174. Bohn RE (2005) From art to science in manufacturing: the evolution of technological knowledge Foundations and Trends in Technology, Information. Opera Manag 1:1-82

175. Haddad FS (2017) Evolving techniques: the need for better technology. Bone Joint J 99:145-146

176. Konyves A, Willis-Owen CA, Spriggins AJ (2010) The long-term benefit of computer-assisted surgical navigation in unicompartmental knee arthroplasty. J Orthop Surg Res 31:94

177. Perlick L, Bathis $H$, Tingart $M$ et al (2004) Minimally invasive unicompartmental knee replacement with a nonimage-based navigation system. Int Orthop 28:193-197

178. Saragaglia D, Picard F, Chaussard C et al (2001) Mise en place des prothe 'ses totales du genou assiste'e par ordinateur: comparaison avec la technique conventionnelle. Rev Chir Orthop. 87:18-28
179. Cobb J, Henckel J, Gomes P et al (2006) Hands-on robotic unicompartmental knee replacement: a prospective, randomised controlled study of the acrobot system. J Bone Joint Surg (Br) 88:188-197

180. Bell SW, Anthony I, Jones B et al (2016) Improved accuracy of component positioning with robotic-assisted unicompartmental knee arthroplasty: data from a prospective, randomized controlled study. J Bone Joint Surg (Am) 98:627-635

181. Herry Y, Batailler C, Lording $T$ et al (2017) Improved joint-line restitution in unicompartmental knee arthroplasty using a robotic-assisted surgical technique. Int Orthop 41:2265-2271

182. van den Heever DJ, Scheffer C, Erasmus P, Dillon E (2011) Contact stresses in a patient-specific unicompartmental knee replacement. Clin Biomech (Bristol, Avon). 26(2):159-166. https://doi.org/10.1016/j.clinb iomech.2010.09.007 (Epub 2010 Oct 14)

183. Jaffry Z, Masjedi M, Clarke S et al (2014) Unicompartmental knee arthroplasties: robot vs patient specific instrumentation. Knee 21(02):428-434

184. Ollivier M, Parratte S, Lunebourg A, Viehweger E, Argenson JN (2016) The John Insall Award: no functional benefit after unicompartmental knee arthroplasty performed with patient-specific instrumentation: a randomized trial. Clin Orthop Relat Res 474(01):60-68

185. Kerens B, Schotanus MG, Boonen B, Kort NP (2015) No radiographic difference between patient-specific guiding and conventional Oxford UKA surgery. Knee Surg Sports Traumatol Arthrosc 23(5):1324-1329. https://doi.org/10.1007/s00167-014-2849-0

186. Moschetti WE, Konopka JF, Rubash HE et al (2016) Can robot-assisted unicompartmental knee arthroplasty be cost-effective? A Markov decision analysis. J Arthroplasty 31:759-765

\section{Publisher's Note}

Springer Nature remains neutral with regard to jurisdictional claims in published maps and institutional affiliations.

\section{Submit your manuscript to a SpringerOpen ${ }^{\circ}$ journal and benefit from:}

- Convenient online submission

- Rigorous peer review

- Open access: articles freely available online

- High visibility within the field

Retaining the copyright to your article

Submit your next manuscript at springeropen.com 